JOURNAL OF INTEGRAL EQUATIONS AND APPLICATIONS

Volume 11, Number 3, Fall 1999

\title{
ON THE ANALYTICITY OF THE CAUCHY INTEGRAL IN SCHAUDER SPACES
}

\author{
MASSIMO LANZA DE CRISTOFORIS AND LUCA PRECISO
}

\begin{abstract}
As is well known, if the contour of integration and the density function belong to a suitable Schauder space, the Cauchy integral belongs to the same Schauder space. We analyze, in this Schauder space setting, the dependence of the Cauchy integral upon its contour and its density function, which we think of as functional variables, and we prove a result of complex analyticity for such dependence. We prove our statement by constructing a functional equation which involves the Cauchy integral, the contour of integration and the density function and by applying to such functional equation the implicit function theorem in its formulation for nonlinear maps between Banach spaces.
\end{abstract}

1. Introduction. In this paper we analyze the analytic dependence of the Cauchy integral

$$
C[\phi, f](\cdot) \equiv \frac{1}{2 \pi i} \text { p.v. } \int_{\partial \mathbf{D}} \frac{f(t) \phi^{\prime}(t)}{\phi(t)-\phi(\cdot)} d t
$$

upon the plane oriented simple closed curve $\phi$ and the density function $f$, both defined on the counterclockwise oriented boundary $\partial \mathbf{D}$ of the plane unit disk $\mathbf{D}$. We assume that both $\phi$ and $f$ belong to a Schauder space, say $\mathcal{C}_{*}^{m, \alpha}(\partial \mathbf{D}, \mathbf{C})$, of complex valued functions of class $\mathcal{C}^{m, \alpha}$ on $\partial \mathbf{D}$, with $m$ a positive natural number and $\alpha \in] 0,1[$. (The ' $*$ ' subscript just means that we are taking the derivatives with respect to the variable on $\partial \mathbf{D}$.) As is well known, under such conditions on $\phi$ and $f$, the function $C[\phi, f](\cdot)$ is also of class $\mathcal{C}_{*}^{m, \alpha}(\partial \mathbf{D}, \mathbf{C})$, and we consider $\mathcal{C}_{*}^{m, \alpha}(\partial \mathbf{D}, \mathbf{C})$ as the target space of $C[\phi, f]$. Although the linear operator $C[\phi, \cdot]$ for a fixed $\phi$ has been studied extensively during the last century and a considerable amount of work has been done on the numerical computation of $C[\phi, f]$, especially in view of the several applications to integral equations and to boundary value problems, the analysis of the nonlinear functional dependence of $C[\phi, f]$

\footnotetext{
Received by the editors on June 23, 1998, and in revised form on November 17 , 1998.

Copyright (C)1999 Rocky Mountain Mathematics Consortium
} 
upon both of its arguments, and in particular on $\phi$, seems to be a subject analyzed only more recently. We mention the contribution of Calderón, Coifman, Meyer, McIntosh, David, whose work implies the analyticity of singular integral operators strictly related to $C$. Calderón [2, Theorem 1] has shown that if $\phi$ is the graph of a Lipschitz function $\psi$, i.e., if $\phi(x)=x+i \psi(x)$ with $\psi^{\prime} \in L^{\infty}(\mathbf{R})$, and if $\left\|\psi^{\prime}\right\|_{L^{\infty}(\mathbf{R})}<\varepsilon$ for some $\varepsilon>0$, then the linear integral operator with singular kernel $\phi^{\prime}(y) /(\phi(y)-\phi(x))$ is an element of the space $\mathcal{L}\left(L^{2}(\mathbf{R}, \mathbf{C}), L^{2}(\mathbf{R}, \mathbf{C})\right)$ of the linear and continuous operators of $L^{2}(\mathbf{R}, \mathbf{C})$ to itself. Then, by using a standard argument of truncated kernels, one can deduce the analytic dependence of the operator with kernel $\phi^{\prime}(y) /(\phi(y)-\phi(x))$ upon $\psi^{\prime}$ when $\left\|\psi^{\prime}\right\|_{L^{\infty}(\mathbf{R})}<\min \{1, \varepsilon\}$, cf., e.g., Meyer and Coifman [19, p. 438]. Later, Coifman, McIntosh and Meyer [3, Theorem 1] and, by different methods, David [6, p. 178], have extended the validity of the same analyticity result to the case in which $\left\|\psi^{\prime}\right\|_{L^{\infty}(\mathbf{R})}<$ 1. Coifman and Meyer [5] have considered the dependence of the Cauchy integral upon an arc-length parametrized curve $\phi$ defined on $\mathbf{R}$, with values in the plane and determined by a function, say $\theta$, which represents the direction of $\phi^{\prime}$, and have shown that the Cauchy operator of $\mathcal{L}\left(L^{2}(\mathbf{R}, \mathbf{C}), L^{2}(\mathbf{R}, \mathbf{C})\right)$ with kernel $\phi^{\prime}(y) /(\phi(y)-\phi(x))$ depends analytically on $\theta$, if $\theta$ ranges in a suitable open subset of the JohnNirenberg space BMO of functions with bounded mean oscillation, cf., Coifman and Meyer [5, p. 10]. Later Wu [25, p. 1310], under the advice of Coifman, has extended the analyticity result of Coifman and Meyer [5] on the Cauchy integral to arc-length parametrized simple closed curves.

In our work, we consider simple closed curves $\phi$, which are not necessarily arc-length parametrized, but which are more regular than those considered by Coifman and Meyer [5]. Correspondingly, the Cauchy operator $C[\phi, \cdot]$ acts in $\mathcal{C}_{*}^{m, \alpha}(\partial \mathbf{D}, \mathbf{C})$, as opposed to that of Coifman and Meyer [5] or Wu [25], which acts in $L^{2}(\mathbf{R}, \mathbf{C})$ or in $L^{2}(\partial \mathbf{D}, \mathbf{C})$, respectively. Although our curves are more regular, our analyticity results cannot be deduced by the work of the authors mentioned above and do not seem to follow by an immediate modification of their methods. An advantage of considering arbitrary parametrizations is that the Cauchy integral $C[\phi, f]$ is defined for $\phi$ in an open subset of $\mathcal{C}_{*}^{m, \alpha}(\partial \mathbf{D}, \mathbf{C})$.

We present an alternative approach to the study of the regularity of the Cauchy integral. As in [15], we represent a Jordan domain 
by an injective and differentiable function, which we denote by $\Psi$, of cl $\mathbf{D}$ to $\mathbf{R}^{2}$, rather than by the more traditional curve $\phi$ parametrizing the boundary of the Jordan domain. Then we observe that a Cauchy integral on a contour is uniquely determined by the pair, say $\left(S^{+}, S^{-}\right)$, of "sectionally holomorphic" functions, which are associated to the Cauchy integral, which are defined in the interior and in the exterior of the contour, respectively, and which satisfy a certain boundary value problem. Then we transform such boundary value problem into a nonlinear boundary value problem of elliptic nature defined on the unit disk, which we now write in the form of an abstract nonlinear operator equation as

$$
\Lambda\left[\Psi, f, T^{+}, \tilde{T}\right]=0,
$$

where $\left(T^{+}, \tilde{T}\right)$ is a pair of functions which is associated to the pair $\left(S^{+}, S^{-}\right)$. Then we show that we can apply to equation (1.2) the implicit function theorem and we deduce that the solution set of (1.2) is the graph of a real analytic operator depending on $(\Psi, f)$. By this result, the real analytic dependence of $C[\phi, f]$ on $(\phi, f)$ follows easily. An advantage of such an approach is that in equation (1.2) there are no singular integrals and that the operator $\Lambda$ of equation (1.2) is easily seen to be analytic. Although to apply the implicit function theorem we still have to prove an isomorphism theorem for the linearized problem associated to (1.2), the difficulties we encounter in doing so are only of linear type. In principle, it seems that our method could be employed even with weaker regularity assumptions on the curve $\phi$ and on the density $f$. Once the real analyticity of $C$ is established, we compute all order derivatives of $C$ and we show that $C$ is actually complex analytic. The statement concerning the real analyticity of $C[\phi, f]$ as a function of $(\phi, f)$ we prove in this paper, finds application in problems of nonlinear integral equations, and in particular in those of a perturbation nature, cf. Lanza and Rogosin [17].

The paper is organized as follows. Section 2 is a section of preliminaries. In Section 3 we show that $C$ is real analytic. In Section 4 we compute the derivatives of $C$ and we show that $C$ is complex analytic.

2. Preliminaries and notation. Let $\mathcal{X}, \mathcal{Y}$ be normed spaces over $\mathbf{K}$, with $\mathbf{K}=\mathbf{R}$ or $\mathbf{K}=\mathbf{C}$. We say that $\mathcal{X}$ is continuously imbedded in $\mathcal{Y}$, provided that $\mathcal{X} \subseteq \mathcal{Y}$ and that the inclusion map is continuous. 
$\mathcal{L}_{\mathbf{K}}(\mathcal{X}, \mathcal{Y})$ denotes the normed space of the continuous $\mathbf{K}$-linear maps of $\mathcal{X}$ into $\mathcal{Y}$ and is equipped with the topology of the uniform convergence on the unit sphere of $\mathcal{X}$. For standard definitions of calculus in normed spaces, we refer, e.g., to Prodi and Ambrosetti [21] or to Berger [1]. Unless otherwise specified, we understand that a finite product of normed spaces is equipped with the sup-norm of the norms of the components. Let $\mathbf{N}$ be the set of nonnegative integers including 0 , and let $n \in \mathbf{N} \backslash\{0\}$. Let $[\cdot]^{n}$ denote the diagonal map of $\mathcal{X}$ to $\mathcal{X}^{n}$ defined by $[v]^{n} \equiv(v, \ldots, v)$ for all $v \in \mathcal{X}$. A complex normed space can be viewed naturally as a real normed space. Accordingly, we will say that a certain map between complex normed spaces is real differentiable, real analytic or real linear, to indicate that such a map is differentiable, analytic or linear, respectively, as a map between the corresponding underlying real spaces. To emphasize that we are retaining the complex structure, we will say that the map is complex differentiable, complex analytic or complex linear. To emphasize that the variables of a certain operator $\mathbf{F}$ are functions rather than scalars, we write $\mathbf{F}[\phi]$ or $\mathbf{F}[\phi, f]$ instead of $\mathbf{F}(\phi)$ or $\mathbf{F}(\phi, f)$. The inverse function of a function $f$ is denoted $f^{(-1)}$ as opposed to the reciprocal of a complex valued function $G$ or the inverse of a matrix $A$, which are denoted $G^{-1}$ and $A^{-1}$, respectively. Let $r \in \mathbf{N} \backslash\{0\} . M_{r}(\mathbf{K})$ denotes the set of $r \times r$ matrices with entries in $\mathbf{K}$. A dot '.' denotes the matrix product. Throughout the paper, we make no formal distinction between complex numbers and pairs of real numbers, so $\mathbf{D}$ denotes the open unit disk both in $\mathbf{C}$ and in $\mathbf{R}^{2}$. Let $B \subseteq \mathbf{R}^{n}$. Then cl $B$ denotes the closure of $B$ and int $B$ denotes the interior of $B$.

We now introduce the Schauder spaces on open subsets of $\mathbf{R}^{n}$. Let $\Omega$ be an open subset of $\mathbf{R}^{n}$, and let $m \in \mathbf{N}$. We denote by $\mathcal{C}^{m}(\Omega, \mathbf{C})$ the space of $m$-times continuously real differentiable complex-valued functions on $\Omega$ and by $\mathcal{C}^{m}(\mathrm{cl} \Omega, \mathbf{C})$ the subspace of those functions $f \in \mathcal{C}^{m}(\Omega, \mathbf{C})$ such that for all $\eta \in \mathbf{N}^{n}$ with $|\eta| \equiv \eta_{1}+\cdots+\eta_{n} \leq m$, the function $D^{\eta} f \equiv \partial^{|\eta|} f /\left(\partial_{1}^{\eta_{1}} \cdots \partial_{n}^{\eta_{n}}\right)$ can be extended with continuity to $\mathrm{cl} \Omega$. In particular, the elements of $\mathcal{C}^{m}(\operatorname{cl} \Omega, \mathbf{C})$ or of $\mathcal{C}^{m}(\Omega, \mathbf{C})$ are not necessarily holomorphic in $\Omega$ even when $m>0, n=2$. If $\Omega$ is a bounded open subset of $\mathbf{R}^{n}$, we equip $\mathcal{C}^{m}(\operatorname{cl} \Omega, \mathbf{C})$ with the norm $\|f\|_{\mathcal{C}^{m}(\operatorname{cl} \Omega, \mathbf{C})} \equiv \sum_{|\eta| \leq m} \sup _{\mathrm{cl} \Omega}\left|D^{\eta} f\right|$. The subspace of $\mathcal{C}^{m}(\operatorname{cl} \Omega, \mathbf{C})$ whose functions have $m$-th order derivatives that are Hölder continuous with exponent $\alpha \in] 0,1]$ is denoted by $\mathcal{C}^{m, \alpha}(\operatorname{cl} \Omega, \mathbf{C})$. If 
$f \in \mathcal{C}^{0, \alpha}(\operatorname{cl} \Omega, \mathbf{C})$, then its Hölder quotient $|f: \Omega|_{\alpha}$ or more simply $|f|_{\alpha}$, is defined as $\sup \left\{\left(\left|f\left(\xi_{1}\right)-f\left(\xi_{2}\right)\right| /\left|\xi_{1}-\xi_{2}\right|^{\alpha}\right): \xi_{1}, \xi_{2} \in \operatorname{cl} \Omega, \xi_{1} \neq \xi_{2}\right\}$. The space $\mathcal{C}^{m, \alpha}(\operatorname{cl} \Omega, \mathbf{C})$ is equipped with its usual norm $\|f\|_{\mathcal{C}^{m, \alpha}(\operatorname{cl} \Omega, \mathbf{C})} \equiv$ $\|f\|_{\mathcal{C}^{m}(\operatorname{cl} \Omega, \mathbf{C})}+\sum_{|\eta|=m}\left|D^{\eta} f\right|_{\alpha}$. It is well known that $\left(\mathcal{C}^{m}(\operatorname{cl} \Omega, \mathbf{C})\right.$, \|\|$\left._{\mathcal{C}^{m}(\operatorname{cl} \Omega, \mathbf{C})}\right)$ and $\left(\mathcal{C}^{m, \alpha}(\operatorname{cl} \Omega, \mathbf{C}),\|\|_{\mathcal{C}^{m, \alpha}(\operatorname{cl} \Omega, \mathbf{C})}\right)$ are complex Banach spaces. Let $r \in \mathbf{N} \backslash\{0\}$. We identify $\mathcal{C}^{m}\left(\operatorname{cl} \Omega, \mathbf{C}^{r}\right), \mathcal{C}^{m, \alpha}\left(\operatorname{cl} \Omega, \mathbf{C}^{r}\right)$ and $\mathcal{C}^{m, \alpha}\left(\operatorname{cl} \Omega, M_{r}(\mathbf{C})\right)$ with the complex Banach spaces $\left(\mathcal{C}^{m}(\operatorname{cl} \Omega, \mathbf{C})\right)^{r}$, $\left(\mathcal{C}^{m, \alpha}(\operatorname{cl} \Omega, \mathbf{C})\right)^{r}$ and $\left(\mathcal{C}^{m, \alpha}(\operatorname{cl} \Omega, \mathbf{C})\right)^{r^{2}}$, respectively. If $B \subseteq \mathbf{C}^{r}$, $\mathcal{C}^{m}(\operatorname{cl} \Omega, B)$ denotes the set $\left\{f \in \mathcal{C}^{m}\left(\operatorname{cl} \Omega, \mathbf{C}^{r}\right): f(\operatorname{cl} \Omega) \subseteq B\right\}$. Similarly we define $\mathcal{C}^{m, \alpha}(\operatorname{cl} \Omega, B)$. Clearly, $\mathcal{C}^{m}\left(\operatorname{cl} \Omega, \mathbf{R}^{r}\right), \mathcal{C}^{m, \alpha}\left(\operatorname{cl} \Omega, \mathbf{R}^{r}\right)$ and $\mathcal{C}^{m, \alpha}\left(\operatorname{cl} \Omega, M_{r}(\mathbf{R})\right)$ are real Banach spaces. In accordance with our definitions, the real Banach spaces $\mathcal{C}^{m, \alpha}(\operatorname{cl} \Omega, \mathbf{C})$ and $\mathcal{C}^{m, \alpha}\left(\operatorname{cl} \Omega, \mathbf{R}^{2}\right)$ coincide algebraically and have equivalent norms. If $n=2$ and if $G \equiv G_{1}+$ $i G_{2} \in \mathcal{C}^{1}(\mathrm{cl} \Omega, \mathbf{C})$, we set as usual $\bar{\partial} G \equiv\left(\partial_{x_{1}} G+i \partial_{x_{2}} G\right) / 2=\left[\left(\partial_{x_{1}} G_{1}-\right.\right.$ $\left.\left.\partial_{x_{2}} G_{2}\right)+i\left(\partial_{x_{1}} G_{2}+\partial_{x_{2}} G_{1}\right)\right] / 2$. Let $\Omega$ be an open bounded connected subset of $\mathbf{R}^{n}$. We set $c[\Omega] \equiv \sup \{(\lambda(x, y) /|x-y|): x, y \in \Omega, x \neq y\}$, where $\lambda(x, y) \equiv \inf \left\{\right.$ length of $\left.\gamma \in \mathcal{C}^{1}([0,1], \Omega): \gamma(0)=x, \gamma(1)=y\right\}$. The open subset $\Omega$ of $\mathbf{R}^{n}$ is said to be regular in the sense of Whitney if $\Omega$ is bounded, connected, and if $c[\Omega]<+\infty$. It is well known that if $\Omega$ is a bounded, connected, open subset of $\mathbf{R}^{n}$ of class $\mathcal{C}^{1}$, then $c[\Omega]<+\infty$, cf., e.g., Jones [10, p. 73].

We now state two abstract results that we need in order to prove some technical facts on the composition and on the reciprocal operator in Schauder spaces. The validity of the following has been pointed out in [14, Proposition 3.11].

Lemma 2.1. Let $\mathcal{X}, \mathcal{Y}, \mathcal{Z}$ be normed spaces. Let $\mathcal{A} \subseteq \mathcal{X}$. Let $\mathbf{S}$ be a map of $\mathcal{Y} \times \mathcal{A}$ to $\mathcal{Z}$ such that, for all $x \in \mathcal{A}$, the map $\mathbf{S}[\cdot, x]$ is linear from $\mathcal{Y}$ to $\mathcal{Z}$, i.e., $\mathbf{S}$ is linear in its first variable. Then the following statements are equivalent.

(i) $\mathbf{S}$ maps bounded sets of $\mathcal{Y} \times \mathcal{A}$ to bounded sets of $\mathcal{Z}$.

(ii) There exists an increasing function $\psi$, i.e., $\psi\left(\rho_{1}\right) \leq \psi\left(\rho_{2}\right)$ whenever $\rho_{1} \leq \rho_{2}$, of $\left[0, \infty\left[\right.\right.$ to itself such that $\|\mathbf{S}[y, x]\|_{\mathcal{Z}} \leq\|y\|_{\mathcal{Y}} \psi\left(\|x\|_{\mathcal{X}}\right)$ for all $(y, x) \in \mathcal{Y} \times \mathcal{A}$.

Proof. Statement (ii) follows by statement (i) by setting $\psi(r) \equiv$ $\sup \left\{\|\mathbf{S}[y, x]\|_{\mathcal{Z}}:(y, x) \in \mathcal{Y} \times \mathcal{A},\|y\|_{\mathcal{Y}}=1,\|x\|_{\mathcal{X}} \leq r\right\}$ where $\sup \varnothing \equiv 0$. 
Statement (i) is an obvious consequence of statement (ii).

The validity of the following abstract proposition concerning the regularity of the reciprocal map is well known and can be verified by a standard argument, cf., e.g., Hille and Phillips [8, Theorems 4.3.2 and 4.3.4].

Proposition 2.2. Let $\mathcal{X}$ be a real or complex Banach algebra with unity, possibly noncommutative. Let $\mathcal{I}$ be the subset of the elements of $\mathcal{X}$ which are invertible with respect to the product of $\mathcal{X}$. Then $\mathcal{I}$ is open and the reciprocal map, which takes an element $x$ of $\mathcal{I}$ to its reciprocal with respect to the product of $\mathcal{X}$, is analytic.

Then we have the following.

Lemma 2.3. Let $m, n, r, h \in \mathbf{N}, n, r, h \geq 1, \alpha, \beta \in] 0,1]$. Let $\Omega$ be an open subset of $\mathbf{R}^{n}$, regular in the sense of Whitney. Then we have the following.

(i) $\mathcal{C}^{m+1}(\operatorname{cl} \Omega, \mathbf{C})$ is continuously imbedded in $\mathcal{C}^{m, \alpha}(\operatorname{cl} \Omega, \mathbf{C})$.

(ii) The pointwise product in $\mathcal{C}^{m, \alpha}(\operatorname{cl} \Omega, \mathbf{R})$ is continuous and $\mathcal{C}^{m, \alpha}(\operatorname{cl} \Omega, \mathbf{R})$ with this product is a commutative Banach algebra with unity.

(iii) The pointwise matrix product in $\mathcal{C}^{m, \alpha}\left(\operatorname{cl} \Omega, M_{r}(\mathbf{R})\right)$ is continuous and $\mathcal{C}^{m, \alpha}\left(\operatorname{cl} \Omega, M_{r}(\mathbf{R})\right)$ with this product is a noncommutative Banach algebra with unity.

(iv) The reciprocal map in $\mathcal{C}^{m, \alpha}\left(\operatorname{cl} \Omega, M_{r}(\mathbf{R})\right)$, which maps an invertible matrix of functions $M$ to its inverse matrix $M^{-1}$, is real analytic from the open subset $\left\{M \in \mathcal{C}^{m, \alpha}\left(\operatorname{cl} \Omega, M_{r}(\mathbf{R})\right): \operatorname{det}(M(x)) \neq 0\right.$, for all $x \in \operatorname{cl} \Omega\}$ of $\mathcal{C}^{m, \alpha}\left(\operatorname{cl} \Omega, M_{r}(\mathbf{R})\right)$ to itself.

(v) Let $\Omega_{1}$ be an open subset of $\mathbf{R}^{r}$, regular in the sense of Whitney. If $F \in \mathcal{C}^{m, \alpha}\left(\operatorname{cl} \Omega_{1}, \mathbf{R}^{h}\right)$ and if $G \in \mathcal{C}^{m, \beta}\left(\operatorname{cl} \Omega, \operatorname{cl} \Omega_{1}\right)$, then $F \circ G \in$ $\mathcal{C}^{m, \gamma_{m}(\alpha, \beta)}\left(\operatorname{cl} \Omega, \mathbf{R}^{h}\right)$, with $\gamma_{0}(\alpha, \beta)=\alpha \beta$ and $\gamma_{m}(\alpha, \beta)=\min \{\alpha, \beta\}$ if $m>0$. Furthermore, there exists an increasing function $\psi$ of $[0,+\infty[$ to itself such that

$$
\|F \circ G\|_{\mathcal{C}^{m, \gamma_{m}(\alpha, \beta)}\left(\operatorname{cl} \Omega, \mathbf{R}^{h}\right)} \leq\|F\|_{\mathcal{C}^{m, \alpha}\left(\operatorname{cl} \Omega_{1}, \mathbf{R}^{h}\right)} \psi\left(\|G\|_{\mathcal{C}^{m, \beta}\left(\operatorname{cl} \Omega, \mathbf{R}^{r}\right)}\right),
$$


for all $(F, G) \in \mathcal{C}^{m, \alpha}\left(\operatorname{cl} \Omega_{1}, \mathbf{R}^{h}\right) \times \mathcal{C}^{m, \beta}\left(\operatorname{cl} \Omega, \operatorname{cl} \Omega_{1}\right)$.

(vi) Let $m \geq 1$. If $G \in \mathcal{C}^{m, \alpha}\left(\operatorname{cl} \Omega, \mathbf{R}^{n}\right)$ is injective and satisfies the condition $\operatorname{det} D G(x) \neq 0$ for all $x$ in $\mathrm{cl} \Omega$, then $G(\Omega)$ is a bounded connected open subset of $\mathbf{R}^{n}, G(\operatorname{cl} \Omega)=\operatorname{cl} G(\Omega), c[G(\Omega)]<+\infty$ and $G^{(-1)} \in \mathcal{C}^{m, \alpha}(\operatorname{cl} G(\Omega), \operatorname{cl} \Omega)$.

Proof. Statement (i) is an obvious consequence of the inclusion $\mathcal{C}^{1}(\operatorname{cl} \Omega, \mathbf{C}) \subseteq \mathcal{C}^{0, \alpha}(\operatorname{cl} \Omega, \mathbf{C})$, which holds because $\Omega$ is regular in the sense of Whitney. Statement (ii) is well known, cf., e.g., [13, Lemma $2.4(\mathrm{v})$ ]; we can prove statement (iii) by using (ii) and by a simple computation. By Proposition 2.2 and by statement (iii) we obtain statement (iv). The first part of statement (v) can be proved by induction on $m$, by using the chain rule and by statement (i) and (ii), see also [13, Lemma 4.20]. We can prove the second part of (v) by Lemma 2.1 and by showing that the composition operator of $\mathcal{C}^{m, \alpha}\left(\operatorname{cl} \Omega_{1}, \mathbf{R}^{h}\right) \times \mathbf{C}^{m, \beta}\left(\operatorname{cl} \Omega, \operatorname{cl} \Omega_{1}\right)$ to $\mathcal{C}^{m, \gamma_{m}(\alpha, \beta)}\left(\operatorname{cl} \Omega, \mathbf{R}^{h}\right)$ maps bounded sequences to bounded sequences, a fact which easily follows by induction on $m$, by the chain rule and by statement (ii). To prove statement (vi), we note that $G(\Omega)$ is open by the inverse function theorem. Since $\operatorname{cl} \Omega$ is compact, $G$ is a homeomorphism of $\operatorname{cl} \Omega$ onto $G(\operatorname{cl} \Omega)$ and we have $G(\operatorname{cl} \Omega)=\operatorname{cl} G(\Omega)$. Inequality $c[G(\Omega)]<+\infty$ follows, for example, from [13, Lemma 4.26]. Then, by induction on $m$, by exploiting statement (v) and equality $D G^{(-1)}(y)=\left(D G\left(G^{(-1)}(y)\right)\right)^{-1}$ for all $y \in \operatorname{cl} G(\Omega)$, we obtain $G^{(-1)} \in \mathcal{C}^{m, \alpha}(\operatorname{cl} G(\Omega), \operatorname{cl} \Omega)$.

As we shall see later, we parametrize Jordan domains by one-to-one functions defined on the unit disk. Thus we will employ the following, cf., [13, Corollary 4.24, Proposition 4.29].

Lemma 2.4. Let $\Psi \in \mathcal{C}^{1}(\mathrm{cl} \mathbf{D}, \mathbf{C})$. Let

$$
\begin{aligned}
l[\Psi] & \equiv \inf \left\{\frac{|\Psi(x)-\Psi(y)|}{|x-y|}: x, y \in \operatorname{cl} \mathbf{D}, x \neq y\right\}, \\
\mathcal{A} & \equiv\left\{\Psi \in \mathcal{C}^{1}(\operatorname{cl} \mathbf{D}, \mathbf{C}): l[\Psi]>0\right\} .
\end{aligned}
$$

Then the following statements hold.

(i) $l[\Psi]>0$ if and only if $\Psi$ is injective and $\operatorname{det} D \Psi(x) \neq 0$ for all $x$ in $\mathrm{cl} \mathbf{D}$. 
(ii) The function of $\mathcal{C}^{1}(\mathrm{cl} \mathbf{D}, \mathbf{C})$ to $\mathbf{R}$ which maps $\Psi$ to $l[\Psi]$ is continuous; in particular, $\mathcal{A}$ is open in $\mathcal{C}^{1}(\mathrm{cl} \mathbf{D}, \mathbf{C})$.

We now want to define the Schauder spaces on plane Jordan curves, which are particular compact subsets of $\mathbf{C}$ with no isolated points. With somewhat more generality, we define the Schauder spaces on a general compact subset $K$ of $C$ with no isolated points. We say that a function $f$ of $K$ to $\mathbf{C}$ is complex differentiable at $z_{0} \in \mathbf{C}$ if $\lim _{K \ni z \rightarrow z_{0}}\left(f(z)-f\left(z_{0}\right)\right) /\left(z-z_{0}\right)$ exists finite. We denote such a limit by $f^{\prime}\left(z_{0}\right)$. As usual, the higher order derivatives, if they exist, are defined inductively. Let $m \in \mathbf{N}$. We denote by $\mathcal{C}_{*}^{m}(K, \mathbf{C})$ the complex normed space of $m$-times continuously complex differentiable functions $f$ of $K$ to $\mathbf{C}$ equipped with the norm $\|f\|_{\mathcal{C}_{*}^{m}(K, \mathbf{C})}=\sum_{i=0}^{m} \sup _{K}\left|f^{(i)}\right|$. We say that $f$ is Hölder continuous on $K$ with exponent $\alpha \in] 0,1]$ provided that $|f: K|_{\alpha} \equiv \sup \left\{\left(\left|f\left(z_{1}\right)-f\left(z_{2}\right)\right| /\left|z_{1}-z_{2}\right|^{\alpha}\right): z_{1}, z_{2} \in K, z_{1} \neq z_{2}\right\}$ is finite. We denote by $\mathcal{C}_{*}^{m, \alpha}(K, \mathbf{C})$ the subspace of $\mathcal{C}_{*}^{m}(K, \mathbf{C})$ of functions having $\alpha$-Hölder continuous $m$-th order derivatives. We equip $\mathcal{C}_{*}^{m, \alpha}(K, \mathbf{C})$ with the norm $\|f\|_{C_{*}^{m, \alpha}(K, \mathbf{C})} \equiv\|f\|_{\mathcal{C}_{*}^{m}(K, \mathbf{C})}+\left|f^{(m)}: K\right|_{\alpha}$. If $B \subseteq \mathbf{C}$, we set $\mathcal{C}_{*}^{m, \alpha}(K, B) \equiv\left\{f \in \mathcal{C}_{*}^{m, \alpha}(K, \mathbf{C}): f(K) \subseteq B\right\}$. Then the following variant of [13, Corollary 4.24, Proposition 4.29] holds.

Lemma 2.5. Let $K$ be a compact subset of $\mathbf{C}$ with no isolated points. Let $\phi \in \mathcal{C}_{*}^{1}(K, \mathbf{C})$. Let

$$
\begin{aligned}
l_{K}[\phi] & \equiv \inf \left\{\frac{|\phi(x)-\phi(y)|}{|x-y|}: x, y \in K, x \neq y\right\} \\
\mathcal{A}_{K} & \equiv\left\{\phi \in \mathcal{C}_{*}^{1}(K, \mathbf{C}): l_{K}[\phi]>0\right\} .
\end{aligned}
$$

Then the following statements hold.

(i) Assume that, for all $\phi \in \mathcal{C}_{*}^{1}(K, \mathbf{C})$ and for all $\tilde{x} \in K$, the limit

$$
\lim _{\left\{(\xi, \eta) \in K^{2}, \xi \neq \eta\right\} \ni(x, y) \rightarrow(\tilde{x}, \tilde{x})} \frac{\phi(x)-\phi(y)}{x-y}
$$

exists and equals $\phi^{\prime}(\tilde{x})$. Then $l_{K}[\phi]>0$ if and only if $\phi$ is injective and $\phi^{\prime}(\xi) \neq 0$ for all $\xi$ in $K$.

(ii) If $K$ is such that $\mathcal{C}_{*}^{1}(K, \mathbf{C})$ is continuously imbedded in $\mathcal{C}_{*}^{0,1}(K, \mathbf{C})$, then the function of $\mathcal{C}_{*}^{1}(K, \mathbf{C})$ to $\mathbf{R}$ which maps $\phi$ to $l_{K}[\phi]$ is continuous and, in particular, $\mathcal{A}_{K}$ is open in $\mathcal{C}_{*}^{1}(K, \mathbf{C})$. 
Proof. The necessity of the condition of statement (i) is obvious. We now show the sufficiency by a contradiction argument, cf. Lanza and Antman [16, Lemma 4.11]. If $l_{K}[\phi]=0$, then by the compactness of $K$, there exist two sequences $\left\{x_{n}\right\}_{n \in \mathbf{N}}$ and $\left\{y_{n}\right\}_{n \in \mathbf{N}}$ in $K$ with $x_{n} \neq y_{n}$ for all $n$, which converge to $\tilde{x}$ and $\tilde{y}$, respectively, and such that

$$
\lim _{n \rightarrow+\infty} \frac{\left|\phi\left(x_{n}\right)-\phi\left(y_{n}\right)\right|}{\left|x_{n}-y_{n}\right|}=0
$$

If $\tilde{x} \neq \tilde{y}$, then $\phi(\tilde{x})=\phi(\tilde{y})$, a contradiction. If $\tilde{x}=\tilde{y}$, then, by the assumption on $K$, we must have $\phi^{\prime}(\tilde{x})=0$, a contradiction. Statement (ii) can be shown by following the proof of the corresponding statement for $\phi \in \mathcal{C}^{1}\left(\operatorname{cl} \Omega, \mathbf{R}^{2}\right)$, with $\Omega$ open and bounded in $\mathbf{R}^{2}$, cf. [13, Proposition 4.29].

Remark 2.6. It can be easily verified that $K=\partial \mathbf{D}$ satisfies the assumptions on $K$ of conditions (i) and (ii) of Lemma 2.5 and that accordingly the conclusions of Lemma 2.5 (i) and (ii) hold for $K=\partial \mathbf{D}$.

We are now ready to state the following, which collects a few facts which we need on the spaces $\mathcal{C}_{*}^{m, \alpha}(K, \mathbf{C})$.

Lemma 2.7. Let $m \in \mathbf{N}, \alpha, \beta \in] 0,1], \phi \in \mathcal{A}_{\partial \mathbf{D}}, L=\phi(\partial \mathbf{D})$. Then the following statements hold.

(i) There exists a positive constant $c_{\phi}$ depending only on $\phi$ such that, for all $f \in \mathcal{C}_{*}^{1}(L, \mathbf{C})$ and for all $z_{1}, z_{2} \in L$,

$$
\left|f\left(z_{1}\right)-f\left(z_{2}\right)\right| \leq c_{\phi}\left(\sup _{L}\left|f^{\prime}\right|\right)\left|z_{1}-z_{2}\right| .
$$

(ii) $\mathcal{C}_{*}^{m+1}(L, \mathbf{C})$ is continuously imbedded in $\mathcal{C}_{*}^{m, \alpha}(L, \mathbf{C})$.

(iii) $\mathcal{C}_{*}^{m}(L, \mathbf{C})$ and $\mathcal{C}_{*}^{m, \alpha}(L, \mathbf{C})$ are complex Banach spaces.

(iv) The pointwise product is continuous in $\mathcal{C}_{*}^{m, \alpha}(L, \mathbf{C})$.

(v) The reciprocal map in $\mathcal{C}_{*}^{m, \alpha}(L, \mathbf{C})$, which maps a nonvanishing function $f$ to its reciprocal, is complex analytic from the open subset $\left\{f \in \mathcal{C}_{*}^{m, \alpha}(L, \mathbf{C}): f(\xi) \neq 0\right.$, for all $\left.\xi \in L\right\}$ of $\mathcal{C}_{*}^{m, \alpha}(L, \mathbf{C})$ to itself. 
(vi) Let $\phi_{1} \in \mathcal{A}_{\partial \mathbf{D}}, L_{1}=\phi_{1}(\partial \mathbf{D})$. If $f \in \mathcal{C}_{*}^{m, \alpha}\left(L_{1}, \mathbf{C}\right)$ and if $g \in \mathcal{C}_{*}^{m, \beta}\left(L, L_{1}\right)$, then $f \circ g \in \mathcal{C}_{*}^{m, \gamma_{m}(\alpha, \beta)}(L, \mathbf{C})$ with $\gamma_{0}(\alpha, \beta)=\alpha \beta$ and $\gamma_{m}(\alpha, \beta)=\min \{\alpha, \beta\}$ if $m>0$. Furthermore, there exists an increasing function $\psi$ of $[0,+\infty[$ to itself such that

$$
\begin{gathered}
\|f \circ g\|_{C_{*}^{m, \gamma_{m}(\alpha, \beta)}(L, \mathbf{C})} \leq\|f\|_{\mathcal{C}_{*}^{m, \alpha}\left(L_{1}, \mathbf{C}\right)} \psi\left(\|g\|_{\mathcal{C}_{*}^{m, \beta}(L, \mathbf{C})}\right), \\
\forall(f, g) \in \mathcal{C}_{*}^{m, \alpha}\left(L_{1}, \mathbf{C}\right) \times \mathcal{C}_{*}^{m, \beta}\left(L, L_{1}\right) .
\end{gathered}
$$

(vii) Let $m \geq 1$. If $g \in \mathcal{C}_{*}^{m, \alpha}(L, \mathbf{C})$ is injective and satisfies condition $g^{\prime}(\xi) \neq 0$ for all $\xi \in L$, then $g^{(-1)} \in \mathcal{C}_{*}^{m, \alpha}(g(L), L)$.

(viii) Let $m \geq 1$ and $\phi \in \mathcal{C}_{*}^{m, \alpha}(\partial \mathbf{D}, \mathbf{C}) \cap \mathcal{A}_{\partial \mathbf{D}}$. Then the map $\mathbf{T}_{\phi}$ defined by $\mathbf{T}_{\phi}[f] \equiv f \circ \phi$ for all $f \in \mathcal{C}_{*}^{m, \alpha}(L, \mathbf{C})$ is a complex linear homeomorphism of $\mathcal{C}_{*}^{m, \alpha}(L, \mathbf{C})$ onto $\mathcal{C}_{*}^{m, \alpha}(\partial \mathbf{D}, \mathbf{C})$.

Proof. We prove (i). Let $j=1,2, \theta_{j} \in[0,2 \pi], s_{j}=e^{i \theta_{j}}$, $z_{j}=\phi\left(s_{j}\right), \sigma\left(\theta_{1}, \theta_{2}\right) \equiv \min \left\{\left|t_{1}-t_{2}\right|: t_{l} \in \mathbf{R}, e^{i t_{l}}=e^{i \theta_{l}}, l=1,2\right\}$, $\eta=\inf \left\{\left(\left|e^{i \theta_{1}}-e^{i \theta_{2}}\right| / \sigma\left(\theta_{1}, \theta_{2}\right): \theta_{1}, \theta_{2} \in[0,2 \pi], \sigma\left(\theta_{1}, \theta_{2}\right) \neq 0\right\}\right.$. As shown in Lanza and Antman [16, Lemma 4.11], $\eta>0$. Since $f \circ \phi\left(e^{i t}\right) \in$ $\mathcal{C}^{1}(\mathbf{R}, \mathbf{C})$, we have

$$
\begin{aligned}
\left|f\left(z_{1}\right)-f\left(z_{2}\right)\right| & \leq\left(\sup _{\theta \in[0,2 \pi]}\left|f^{\prime}\left(\phi\left(e^{i \theta}\right)\right) \phi^{\prime}\left(e^{i \theta}\right)\right|\right) \sigma\left(\theta_{1}, \theta_{2}\right) \\
& \leq\left(\sup _{L}\left|f^{\prime}\right|\right)\left(\sup _{[0,2 \pi]}\left|\phi^{\prime}\right|\right) \eta^{-1}\left(l_{\partial \mathbf{D}}[\phi]\right)^{-1}\left|z_{1}-z_{2}\right| .
\end{aligned}
$$

Then statement (i) follows by setting $c_{\phi} \equiv\left(\sup _{[0,2 \pi]}\left|\phi^{\prime}\right|\right) \eta^{-1}\left(l_{\partial \mathbf{D}}[\phi]\right)^{-1}$. Statement (ii) is an immediate consequence of (i). We now prove statement (iii). It clearly suffices to show that $\mathcal{C}_{*}^{m}(L, \mathbf{C})$ is complete. We proceed by induction on $m$. Case $m=0$ is well known. Case $m=1$ can be shown by observing that, if $f \in \mathcal{C}_{*}^{1}(L, \mathbf{C})$, then $f\left(\phi\left(e^{i t}\right)\right) \in \mathcal{C}^{1}(\mathbf{R}, \mathbf{C})$ and by using a standard argument. Case $m+1$ can be deduced by case $m$ and by applying case $m=1$. Statement (iv) can be proved by a standard inductive argument, cf., e.g., [13, Lemma 2.4(v)] and by using statement (ii). Statement (v) is an immediate consequence of (iii), (iv) and of Proposition 2.2. We can prove statement (vii) and the first part of (vi) by induction on $m$, by using the chain rule, the rule of differentiation of the inverses and 
statements (ii) and (iv). By statements (ii) and (iv) and, by induction on $m$, it can be easily shown that the composition operator maps bounded sequences of $\mathcal{C}_{*}^{m, \alpha}\left(L_{1}, \mathbf{C}\right) \times \mathcal{C}_{*}^{m, \beta}\left(L, L_{1}\right)$ to bounded sequences of $\mathcal{C}_{*}^{m, \gamma_{m}(\alpha, \beta)}(L, \mathbf{C})$. Then, by Lemma 2.1, we conclude the existence of $\psi$ as in the second part of statement (vi). Statement (viii) is an immediate consequence of statements (vi) and (vii).

Now let $\phi \in \mathcal{A}_{\partial \mathbf{D}}$. By the Jordan theorem, cf., e.g., Godbillon [7, Corollary 4.4, p. 214], $\mathbf{C} \backslash \phi(\partial \mathbf{D})$ consists of two open connected components. We denote by $\mathbf{I}[\phi]$ and $\mathbf{E}[\phi]$ the bounded and the unbounded components of $\mathbf{C} \backslash \phi(\partial \mathbf{D})$, respectively. We collect in the following lemma some properties of $\mathbf{I}[\phi]$, of $\mathbf{E}[\phi]$ and of the trace of a function of class $\mathcal{C}^{m, \alpha}$ in $\operatorname{cl} \mathbf{I}[\phi]$.

Lemma 2.8. The following statements hold.

(i) If $\phi \in \mathcal{A}_{\partial \mathbf{D}}$, then $\partial \mathbf{I}[\phi]=\partial \mathbf{E}[\phi]=\phi(\partial \mathbf{D})$ and $c[\mathbf{I}[\phi]]<+\infty$.

(ii) Let $m \in \mathbf{N}, \alpha \in] 0,1], \phi \in \mathcal{C}_{*}^{m, \alpha}(\partial \mathbf{D}, \mathbf{C}) \cap \mathcal{A}_{\partial \mathbf{D}}$. Then the trace operator $R$ from $\mathcal{C}^{m, \alpha}(\operatorname{cl} \mathbf{I}[\phi], \mathbf{C})$ to $\mathcal{C}_{*}^{m, \alpha}(\phi(\partial \mathbf{D}), \mathbf{C})$ defined by $R[F]=F_{/ \phi(\partial \mathbf{D})}$ is complex linear and continuous.

Proof. Conditions $\phi\left(e^{i t}\right) \in \mathcal{C}^{1}(\mathbf{R}, \mathbf{C}), \phi^{\prime}\left(e^{i t}\right) \neq 0$ for all $t \in \mathbf{R}$, $l_{\partial \mathbf{D}}[\phi]>0$ imply that $\phi(\partial \mathbf{D})$ is a real connected submanifold of class $\mathcal{C}^{1}$ and of codimension one of $\mathbf{C}$. It follows that the boundary of $\mathbf{I}[\phi]$ and $\mathbf{E}[\phi]$ is $\phi(\partial \mathbf{D})$ and that $\mathbf{I}[\phi]$ and $\mathbf{E}[\phi]$ are open subsets of $\mathbf{C}$ of class $\mathcal{C}^{1}$. Then $c[\mathbf{I}[\phi]]<+\infty$. We now fix $m$ and prove by induction on $j=0, \ldots, m$, that $R$ is continuous from $\mathcal{C}^{j, \alpha}(\operatorname{cl} \mathbf{I}[\phi], \mathbf{C})$ to $\mathcal{C}_{*}^{j, \alpha}(\phi(\partial \mathbf{D}), \mathbf{C})$. Case $j=0$ is obvious. We now assume that the statement holds for $j \in\{0, \ldots, m-1\}$ and prove it for $j+1$. Let $F \in \mathcal{C}^{j+1, \alpha}(\operatorname{cl} \mathbf{I}[\phi], \mathbf{C})$ and let $\phi_{1}$ and $\phi_{2}$ be the real and the imaginary part, respectively, of $\phi$. Since $\phi\left(e^{i t}\right)$ belongs to $\mathcal{C}^{m, \alpha}([0,2 \pi], \mathbf{C})$ then $\phi_{1}$ and $\phi_{2}$ belong to $\mathcal{C}_{*}^{m, \alpha}(\partial \mathbf{D}, \mathbf{C})$. By Lemma $2.3(\mathrm{v})$, we have $F\left(\phi\left(e^{i t}\right)\right) \in \mathcal{C}^{1}(\mathbf{R}, \mathbf{C})$. Then $F^{\prime}$ exists. By the chain rule applied to the function $F\left(\phi\left(e^{i t}\right)\right)$, we deduce that

$$
\begin{gathered}
F^{\prime}(z)=\left[\frac{\partial F}{\partial x}(z) \phi_{1}^{\prime}\left(\phi^{(-1)}(z)\right)+\frac{\partial F}{\partial y}(z) \phi_{2}^{\prime}\left(\phi^{(-1)}(z)\right)\right]\left(\phi^{\prime}\left(\phi^{(-1)}(z)\right)\right)^{-1}, \\
\forall z \in \phi(\partial \mathbf{D}) .
\end{gathered}
$$


By inductive assumption and by Lemma 2.7 (iv), (v), (vi) and (vii), there exists a positive constant $c$ such that $\left\|F^{\prime}\right\|_{\mathcal{C}_{*}^{j, \alpha}(\phi(\partial \mathbf{D}), \mathbf{C})}$ $\leq c\|F\|_{\mathcal{C}^{j+1, \alpha}(\operatorname{cl} \mathbf{I}[\phi], \mathbf{C})}$. Then statement (ii) follows immediately.

We now show that our representation of a Jordan domain depends analytically on the curve which parametrizes the boundary of the Jordan domain. To do so we need the following which is a restatement of a corresponding lemma of [15, Lemma 2.13].

Lemma 2.9. Let $m \in \mathbf{N} \backslash\{0\}, \alpha \in] 0,1\left[\right.$. Let $\phi_{0} \in \mathcal{C}_{*}^{m, \alpha}(\partial \mathbf{D}, \mathbf{C}) \cap$ $\mathcal{A}_{\partial \mathbf{D}}, z_{0} \in \mathbf{I}\left[\phi_{0}\right]$. Then the following hold.

(i) There exists at least an element $\Psi_{0} \in \mathcal{C}^{m, \alpha}(\mathrm{cl} \mathbf{D}, \mathbf{C}) \cap \mathcal{A}$ such that $\Psi_{0 / \partial \mathbf{D}}=\phi_{0}$ and that $\Psi_{0}(0)=z_{0}$.

(ii) There exists a continuous complex linear extension operator $E$ of $\mathcal{C}_{*}^{m, \alpha}(\partial \mathbf{D}, \mathbf{C})$ to $\mathcal{C}^{m, \alpha}(\mathrm{cl} \mathbf{D}, \mathbf{C})$ such that the affine map between the same spaces defined by

$$
E_{\phi_{0}}[\phi] \equiv \Psi_{0}+E\left[\phi-\phi_{0}\right]
$$

maps an open neighborhood $\mathcal{U}_{\phi_{0}}$ of $\phi_{0}$ contained in $\mathcal{C}_{*}^{m, \alpha}(\partial \mathbf{D}, \mathbf{C}) \cap \mathcal{A}_{\partial \mathbf{D}}$ into $\left\{\Psi \in \mathcal{C}^{m, \alpha}(\mathrm{cl} \mathbf{D}, \mathbf{C}) \cap \mathcal{A}: \Psi(0)=z_{0}\right\}$ and satisfies

$$
E_{\phi_{0}}[\phi]_{/ \partial \mathbf{D}}=\phi, \quad \forall \phi \in \mathcal{U}_{\phi_{0}} .
$$

Proof. Clearly, the function $\phi_{0}\left(e^{i t}\right)$ is a simple closed curve of class $\mathcal{C}^{m, \alpha}$ defined on $[0,2 \pi]$ with $(d / d t)\left\{\phi_{0}\left(e^{i t}\right)\right\} \neq 0$ for all $t \in[0,2 \pi]$. Then, by $[\mathbf{1 5}$, Lemmas 2.7, 2.13 (i)], statement (i) holds. To prove statement (ii), we take $k \in \mathcal{C}^{\infty}([0,1],[0,1])$ such that $k([0,(1 / 3)])=$ $\{0\}, k([(2 / 3), 1])=\{1\}$ and we set $E[h](x) \equiv h(x /|x|) k(|x|)$, for all $x \in \mathrm{cl} \mathbf{D}, h \in \mathcal{C}_{*}^{m, \alpha}(\partial \mathbf{D}, \mathbf{C})$. By exploiting Lemma 2.3 (ii) and (v), it can be verified that $E[\cdot]$ is a complex linear and continuous operator of $\mathcal{C}_{*}^{m, \alpha}(\partial \mathbf{D}, \mathbf{C})$ to $\mathcal{C}^{m, \alpha}(\mathrm{cl} \mathbf{D}, \mathbf{C})$ and that $E[h]_{/ \partial \mathbf{D}}=h$ for all $h \in \mathcal{C}_{*}^{m, \alpha}(\partial \mathbf{D}, \mathbf{C})$. Then, by using Lemmas 2.4 (ii) and 2.5 (ii), it is easy to check that the affine map defined in (2.10) satisfies the required properties. 
3. Introduction of a modified problem and analyticity of the Cauchy integral. We now turn our attention to the dependence of the Cauchy integral of (1.1) upon $\phi, f$. We understand that all line integrals on $\partial \mathbf{D}$ are computed with respect to the parametrization $\theta \mapsto e^{i \theta}, \theta \in[0,2 \pi]$ and that all line integrals on $\phi \in \mathcal{C}_{*}^{1}(\partial \mathbf{D}, \mathbf{C})$ are computed with respect to the parametrization $\theta \mapsto \phi\left(e^{i \theta}\right)$. Let $\phi \in \mathcal{C}_{*}^{1}(\partial \mathbf{D}, \mathbf{C}) \cap \mathcal{A}_{\partial \mathbf{D}}$. We denote by ind $[\phi]$ the index of the curve $\theta \mapsto \phi\left(e^{i \theta}\right), \theta \in[0,2 \pi]$ with respect to any of the points of $\mathbf{I}[\phi]$ :

$$
\text { ind }[\phi] \equiv \frac{1}{2 \pi i} \int_{\phi} \frac{d \xi}{\xi-z}, \quad z \in \mathbf{I}[\phi] .
$$

The map ind $[\cdot]$ is obviously constant on the open connected components of $\mathcal{A}_{\partial \mathbf{D}}$ in $\mathcal{C}_{*}^{1}(\partial \mathbf{D}, \mathbf{C})$. Now it is well known that the Cauchy integral $\int_{\phi} f \circ \phi^{(-1)}(\xi) /(\xi-z) d \xi$ determines a so-called "sectionally holomorphic function" which vanishes at infinity and which jumps across the contour of integration, as shown by the Plemelj formula. Also the jump condition and the condition at infinity determine the "sectionally holomorphic function." We formulate such known facts in the following statement.

Theorem 3.2. Let $m \in \mathbf{N} \backslash\{0\}, \alpha \in] 0,1\left[\right.$. Let $\phi \in \mathcal{C}_{*}^{m, \alpha}(\partial \mathbf{D}, \mathbf{C}) \cap$ $\mathcal{A}_{\partial \mathbf{D}}, f \in \mathcal{C}_{*}^{m, \alpha}(\partial \mathbf{D}, \mathbf{C})$. Then there exists one and only one pair of functions

$$
\left(S^{+}, S^{-}\right) \in \mathcal{C}^{m, \alpha}(\operatorname{cl} \mathbf{I}[\phi], \mathbf{C}) \times\left(\mathcal{C}^{1}(\operatorname{cl} \mathbf{E}[\phi], \mathbf{C}) \cap \mathcal{C}_{*}^{m, \alpha}(\phi(\partial \mathbf{D}), \mathbf{C})\right),
$$

which satisfies the following boundary value problem associated to $(\phi, f)$

$$
\begin{cases}\bar{\partial} S^{+}=0 & \text { in } \mathbf{I}[\phi], \\ \bar{\partial} S^{-}=0 & \text { in } \mathbf{E}[\phi], \\ S^{+}-S^{-}=f \circ \phi^{(-1)} & \text { on } \phi(\partial \mathbf{D}), \\ S^{-}(\infty) \equiv \lim _{z \rightarrow \infty} S^{-}(z)=0 . & \end{cases}
$$

We denote such a unique solution $\left(S^{+}, S^{-}\right)$by $\left(\mathbf{S}^{+}[\phi, f], \mathbf{S}^{-}[\phi, f]\right)$. The functions $\mathbf{S}^{+}[\phi, f]$ and $\mathbf{S}^{-}[\phi, f]$ can be written explicitly as follows

$$
\begin{aligned}
& \mathbf{S}^{+}[\phi, f](z)=\frac{\operatorname{ind}[\phi]}{2 \pi i} \int_{\phi} \frac{f \circ \phi^{(-1)}(\xi)}{\xi-z} d \xi \quad \forall z \in \mathbf{I}[\phi], \\
& \mathbf{S}^{-}[\phi, f](z)=\frac{\operatorname{ind}[\phi]}{2 \pi i} \int_{\phi} \frac{f \circ \phi^{(-1)}(\xi)}{\xi-z} d \xi \quad \forall z \in \mathbf{E}[\phi],
\end{aligned}
$$


and the following Plemelj formula holds

$$
\begin{gathered}
\mathbf{S}^{ \pm}[\phi, f](z)= \pm \frac{1}{2} f \circ \phi^{(-1)}(z)+\frac{\text { ind }[\phi]}{2 \pi i} \text { p.v. } \int_{\phi} \frac{f \circ \phi^{(-1)}(\xi)}{\xi-z} d \xi \\
\forall z \in \phi(\partial \mathbf{D}) .
\end{gathered}
$$

Proof. We first consider the uniqueness. Assume that $\left(S_{j}^{+}, S_{j}^{-}\right)$, $j=1,2$, are solutions of $(3.3)$; then

$$
\begin{cases}\bar{\partial}\left[S_{1}^{+}-S_{2}^{+}\right]=0 & \text { in } \mathbf{I}[\phi], \\ \bar{\partial}\left[S_{1}^{-}-S_{2}^{-}\right]=0 & \text { in } \mathbf{E}[\phi], \\ \left(S_{1}^{+}-S_{2}^{+}\right)-\left(S_{1}^{-}-S_{2}^{-}\right)=0 & \text { on } \phi(\partial \mathbf{D}), \\ \left(S_{1}^{-}-S_{2}^{-}\right)(\infty)=0 . & \end{cases}
$$

We observe that, by Lemma 2.8 (i), we have $\partial \mathbf{I}[\phi]=\partial \mathbf{E}[\phi]=\phi(\partial \mathbf{D})$. Thus the function

$$
G(z)= \begin{cases}\left(S_{1}^{+}-S_{2}^{+}\right)(z) & \text { if } z \in \operatorname{cl} \mathbf{I}[\phi] \\ \left(S_{1}^{-}-S_{2}^{-}\right)(z) & \text { if } z \in \mathbf{C} \backslash \operatorname{cl} \mathbf{I}[\phi]\end{cases}
$$

is holomorphic in $\mathbf{C} \backslash \phi(\partial \mathbf{D})$ and continuous on $\mathbf{C}$. Then a well-known result, cf., e.g., Muskhelishvili [20, p. 36], implies that $G$ is holomorphic in C. Since $G(\infty)=0$, Liouville's theorem implies that $G=0$. By Lemma 2.7 (vi) and (vii), we have $f \circ \phi^{(-1)} \in \mathcal{C}_{*}^{m, \alpha}(\phi(\partial \mathbf{D}), \mathbf{C})$ and thus, by the well-known properties of the Cauchy integral, see, e.g., Lu $[\mathbf{1 8}$, pp. 23, 28, 36] and by Lemma 2.8 (ii), we deduce the existence of $\left(S^{+}, S^{-}\right)$and equation (3.4).

Then, by Lemma 2.8 (ii), we deduce the following corollary.

Corollary 3.5. Let $m \in \mathbf{N} \backslash\{0\}$, and let $\alpha \in] 0,1[$. For all $(\phi, f) \in\left(\mathcal{C}_{*}^{m, \alpha}(\partial \mathbf{D}, \mathbf{C}) \cap \mathcal{A}_{\partial \mathbf{D}}\right) \times \mathcal{C}_{*}^{m, \alpha}(\partial \mathbf{D}, \mathbf{C})$ the integral

$$
\begin{aligned}
C[\phi, f](\cdot) & \equiv \frac{1}{2 \pi i} \text { p.v. } \int_{\partial \mathbf{D}} \frac{f(t) \phi^{\prime}(t)}{\phi(t)-\phi(\cdot)} d t \\
& =\frac{1}{2 \pi i} \text { p.v. } \int_{\phi} \frac{f \circ \phi^{(-1)}(\xi)}{\xi-\phi(\cdot)} d \xi
\end{aligned}
$$


belongs to $\mathcal{C}_{*}^{m, \alpha}(\partial \mathbf{D}, \mathbf{C})$. Thus $C$ defines a nonlinear operator of $\left(\mathcal{C}_{*}^{m, \alpha}(\partial \mathbf{D}, \mathbf{C}) \cap \mathcal{A}_{\partial \mathbf{D}}\right) \times \mathcal{C}_{*}^{m, \alpha}(\partial \mathbf{D}, \mathbf{C})$ to $\mathcal{C}_{*}^{m, \alpha}(\partial \mathbf{D}, \mathbf{C})$.

Remark 3.6. By (3.4), by Lemma 2.8 (ii) and by the constancy of ind [.] on the open connected components of $\mathcal{C}_{*}^{m, \alpha}(\partial \mathbf{D}, \mathbf{C}) \cap \mathcal{A}_{\partial \mathbf{D}}$, the study of the regularity of the operator $C[\phi, f]$ is equivalent to that of the operator from $\left(\mathcal{C}_{*}^{m, \alpha}(\partial \mathbf{D}, \mathbf{C}) \cap \mathcal{A}_{\partial \mathbf{D}}\right) \times \mathcal{C}_{*}^{m, \alpha}(\partial \mathbf{D}, \mathbf{C})$ to $\mathcal{C}_{*}^{m, \alpha}(\partial \mathbf{D}, \mathbf{C})$ which maps a pair $(\phi, f)$ to $\mathbf{S}^{+}[\phi, f] \circ \phi$.

To enable the application of our methods, we now represent the contour of integration of our Cauchy integrals by the restriction to $\partial \mathbf{D}$ of a function $\Psi$ defined on $\mathrm{cl} \mathbf{D}$. Let $\Psi \in \mathcal{C}^{m, \alpha}(\operatorname{cl} \mathbf{D}, \mathbf{C}) \cap \mathcal{A}, m \geq 1$, $\phi \equiv \Psi_{/ \partial \mathbf{D}}$. By Brouwer's theorem on the invariance of the domain, cf., e.g., Hurewicz and Wallman [9, p. 95], and by a simple topological argument, cf., e.g., [15, Lemma 2.2], we have $\Psi(\mathbf{D})=\mathbf{I}[\phi]$ and $\mathbf{C} \backslash \Psi(\operatorname{cl} \mathbf{D})=\mathbf{E}[\phi]$. Now our aim is to prove that the nonlinear operator which takes the pair $(\Psi, f)$ to $\mathbf{S}^{+}[\Psi / \partial \mathbf{D}, f] \circ \Psi$ is real analytic from $\left(\mathcal{C}^{m, \alpha}(\mathrm{cl} \mathbf{D}, \mathbf{C}) \cap \mathcal{A}\right) \times \mathcal{C}_{*}^{m, \alpha}(\partial \mathbf{D}, \mathbf{C})$ to $\mathcal{C}^{m, \alpha}(\mathrm{cl} \mathbf{D}, \mathbf{C})$. As a consequence, we can deduce by Lemmas 2.8 (ii) and 2.9 the real analyticity of the operator $C$.

We note that problem (3.3) has been formulated in part on the unbounded domain $\mathbf{C} \backslash \Psi(\mathrm{cl} \mathbf{D})$. Since we find it more convenient to work on a bounded domain, we now transform the problem in $\mathbf{C} \backslash \Psi(\mathrm{cl} \mathbf{D})$ into a problem defined in a bounded domain. To do so, we need the following.

Lemma 3.7. Let $m \in \mathbf{N} \backslash\{0\}, \alpha \in] 0,1\left[\right.$. Let $\Psi_{0} \in \mathcal{C}^{m, \alpha}(\operatorname{cl} \mathbf{D}, \mathbf{C}) \cap \mathcal{A}$. Then there exists an open neighborhood $\mathcal{W}_{\Psi_{0}}$ of $\Psi_{0}$ in the open subset $\mathcal{C}^{m, \alpha}(\operatorname{cl} \mathbf{D}, \mathbf{C}) \cap \mathcal{A}$ of $\mathcal{C}^{m, \alpha}(\operatorname{cl} \mathbf{D}, \mathbf{C})$ and a complex analytic map $\mathbf{G}$ of $\mathcal{W}_{\Psi_{0}}$ to $\mathcal{C}^{m, \alpha}(\operatorname{cl} \mathbf{D}, \mathbf{C}) \cap \mathcal{A}$ such that

$$
\begin{aligned}
\mathbf{G}[\Psi](t) & =\frac{1}{\Psi(t)-\Psi(0)} \quad \forall t \in \partial \mathbf{D}, \\
\mathbf{G}[\Psi](0) & =0
\end{aligned}
$$

for all $\Psi \in \mathcal{W}_{\Psi_{0}}$.

Proof. By Lemma 2.8 (ii), the map of $\mathcal{C}^{m, \alpha}(\operatorname{cl} \mathbf{D}, \mathbf{C})$ to $\mathcal{C}_{*}^{m, \alpha}(\partial \mathbf{D}, \mathbf{C})$, 
which takes $\Psi$ to $\left(\Psi_{/ \partial \mathbf{D}}-\Psi(0)\right)$, is complex linear and continuous and thus complex analytic. By Lemma 2.7 (v), the reciprocal map is complex analytic from $\mathcal{C}_{*}^{m, \alpha}(\partial \mathbf{D}, \mathbf{C} \backslash\{0\})$ to itself. Since the map $H(z) \equiv 1 /(z-\Psi(0))$ is a one-to-one holomorphic map of $\mathbf{C} \backslash\{\Psi(0)\}$ onto $\mathbf{C} \backslash\{0\}$, we have $1 /\left(\Psi_{/ \partial \mathbf{D}}(\cdot)-\Psi(0)\right) \in \mathcal{C}_{*}^{m, \alpha}(\partial \mathbf{D}, \mathbf{C} \backslash\{0\}) \cap \mathcal{A}_{\partial \mathbf{D}}$ for all $\Psi \in \mathcal{C}^{m, \alpha}(\operatorname{cl} \mathbf{D}, \mathbf{C}) \cap \mathcal{A}$. Then we can conclude the proof by applying Lemma 2.9 , with $\phi_{0}(\cdot) \equiv 1 /\left(\Psi_{0}(\cdot)-\Psi_{0}(0)\right)$ in $\partial \mathbf{D}$ and with $z_{0}=0$.

We now reformulate the boundary value problem (3.3) on a bounded domain.

Proposition 3.8. Let $m \in \mathbf{N} \backslash\{0\}, \alpha \in] 0,1\left[\right.$. Let $\Psi_{0} \in$ $\mathcal{C}^{m, \alpha}(\mathrm{cl} \mathbf{D}, \mathbf{C}) \cap \mathcal{A}$. Let $\mathcal{W}_{\Psi_{0}}$ be the neighborhood of $\Psi_{0}$ in $\mathcal{C}^{m, \alpha}(\operatorname{cl} \mathbf{D}, \mathbf{C}) \cap$ $\mathcal{A}$ of Lemma 3.7. Let $(\Psi, f) \in \mathcal{W}_{\Psi_{0}} \times \mathcal{C}_{*}^{m, \alpha}(\partial \mathbf{D}, \mathbf{C})$. Then the boundary value problem

$$
\begin{cases}\bar{\partial}\left[T^{+} \circ \Psi^{(-1)}\right]=0 & \text { in } \Psi(\mathbf{D}), \\ \bar{\partial}\left[\tilde{T} \circ(\mathbf{G}[\Psi])^{(-1)}\right]=0 & \text { in } \mathbf{G}[\Psi](\mathbf{D}), \\ T^{+}-\tilde{T}=f & \text { on } \partial \mathbf{D}, \\ \tilde{T}(0)=0, & \end{cases}
$$

has a unique solution $\left(T^{+}, \tilde{T}\right) \in\left(\mathcal{C}^{m, \alpha}(\mathrm{cl} \mathbf{D}, \mathbf{C})\right)^{2}$. Such a unique solution is delivered by the formulas

$$
\begin{aligned}
T^{+}(z) & =\mathbf{S}^{+}[\Psi / \partial \mathbf{D}, f](\Psi(z)), \quad \forall z \in \operatorname{cl} \mathbf{D}, \\
\tilde{T}(z) & = \begin{cases}\mathbf{S}^{-}[\Psi / \partial \mathbf{D}, f]\left(\Psi(0)+(\mathbf{G}[\Psi](z))^{-1}\right) & \text { if } z \in \operatorname{cl} \mathbf{D} \backslash\{0\}, \\
\mathbf{S}^{-}\left[\Psi_{/ \partial \mathbf{D}}, f\right](\infty)=0 & \text { if } z=0 .\end{cases}
\end{aligned}
$$

Proof. Assume that $\left(T^{+}, \tilde{T}\right) \in\left(\mathcal{C}^{m, \alpha}(\operatorname{cl} \underset{\tilde{S}}{\mathbf{D}}, \mathbf{C})\right)^{2}$ solves (3.9). Then, by Lemma $2.3(\mathrm{v})$ and (vi), the function $\tilde{S} \equiv \tilde{T} \circ(\mathbf{G}[\Psi])^{(-1)}$ belongs to $\mathcal{C}^{m, \alpha}(\operatorname{cl} \mathbf{G}[\Psi](\mathbf{D}), \mathbf{C})$. We now observe that the function $H(z) \equiv$ $1 /(z-\Psi(0))$ is a one-to-one map of $\mathbf{C} \backslash\{\Psi(0)\}$ onto $\mathbf{C} \backslash\{0\}$ and that it is holomorphic with inverse map $H^{(-1)}(w)=\Psi(0)+(1 / w)$. Furthermore, $H$ is a one-to-one map of $\operatorname{cl}(\mathbf{C} \backslash \Psi(\operatorname{cl} \mathbf{D}))$ onto $(\operatorname{cl} \mathbf{G}[\Psi](\mathbf{D})) \backslash\{0\}$. Then 
by chain rule and Lemmas 2.7 (ii), (vi) and 2.8 (ii), we have $S^{-} \equiv$ $\tilde{S} \circ H \in \mathcal{C}^{1}(\operatorname{cl}(\mathbf{C} \backslash \Psi(\operatorname{cl} \mathbf{D})), \mathbf{C}) \cap \mathcal{C}_{*}^{m, \alpha}(\Psi(\partial \mathbf{D}), \mathbf{C})$ and $S^{-}(\infty)=\tilde{S}(0)=$ $\tilde{T}\left(\mathbf{G}[\Psi]^{(-1)}(0)\right)=\tilde{T}(0)=0$. By Lemma $2.3(\mathrm{v})$ and (vi), we have $S^{+} \equiv T^{+} \circ \Psi^{(-1)} \in \mathcal{C}^{m, \alpha}(\operatorname{cl} \Psi(\mathbf{D}), \mathbf{C})$ and an immediate computation shows that $\left(S^{+}, S^{-}\right)$solves problem (3.3) for $\phi \equiv \Psi_{/ \partial \mathbf{D}}$. Then $S^{+}=\mathbf{S}^{+}\left[\Psi_{/ \partial \mathbf{D}}, f\right], S^{-}=\mathbf{S}^{-}\left[\Psi_{/ \partial \mathbf{D}}, f\right]$. This shows the uniqueness of problem (3.9) and that the formulas for $T^{+}, \tilde{T}$ hold provided that problem (3.9) has at least a solution.

We now prove the existence for problem (3.9). We define the function $\tilde{S}$ from $\mathrm{cl} \mathbf{G}[\Psi](\mathbf{D})$ to $\mathbf{C}$ by setting

$$
\tilde{S}(w) \equiv \begin{cases}\mathbf{S}^{-}[\Psi / \partial \mathbf{D}, f](\Psi(0)+(1 / w)) & \text { if } w \in(\operatorname{cl} \mathbf{G}[\Psi](\mathbf{D})) \backslash\{0\}, \\ \mathbf{S}^{-}\left[\Psi_{/ \partial \mathbf{D}}, f\right](\infty) & \text { if } w=0 .\end{cases}
$$

We set $\gamma \equiv \mathbf{G}[\Psi]_{/ \partial \mathbf{D}}$. By Lemma 2.8 (ii) we have $\gamma \in \mathcal{C}_{*}^{m, \alpha}(\partial \mathbf{D}, \mathbf{C} \backslash\{0\}) \cap$ $\mathcal{A}_{\partial \mathbf{D}}$, and an easy computation shows that

$$
\tilde{S}(w)=-w \cdot \mathbf{S}^{+}\left[\gamma, \frac{f}{\gamma}\right](w)
$$

for all $w \in \operatorname{cl} \mathbf{I}[\gamma]$. Since $\mathbf{S}^{+}[\phi, f] \in \mathcal{C}^{m, \alpha}(\operatorname{cl} \mathbf{I}[\phi], \mathbf{C})$ for all $(\phi, f) \in$ $\left(\mathcal{C}_{*}^{m, \alpha}(\partial \mathbf{D}, \mathbf{C}) \cap \mathcal{A}_{\partial \mathbf{D}}\right) \times \mathcal{C}_{*}^{m, \alpha}(\partial \mathbf{D}, \mathbf{C})$, Lemmas $2.7(\mathrm{iv}),(\mathrm{v})$ and $2.3(\mathrm{i})$ and (ii) imply that $\tilde{S} \in \mathcal{C}^{m, \alpha}(\operatorname{cl} \mathbf{G}[\Psi](\mathbf{D}), \mathbf{C})$. Then, by Lemma 2.3 (v) and (vi), we deduce that $\left(T^{+}, \tilde{T}\right)$ defined as in (3.10) belongs to $\left(\mathcal{C}^{m, \alpha}(\operatorname{cl} \mathbf{D}, \mathbf{C})\right)^{2}$ and solves problem (3.9).

To proceed further, we wish to rewrite the equations of (3.9) in a way suitable to the application of our methods. To do so we introduce the following lemma, whose proof is of immediate verification, cf., [15, Lemma 3.1].

Lemma 3.11. Let $m \in \mathbf{N}, \alpha \in] 0,1]$. Let $\Omega$ be an open subset of $\mathbf{R}^{2}$. Let $\mathbf{L}$ be the linear and continuous map of $\mathcal{C}^{m, \alpha}\left(\operatorname{cl} \Omega, M_{2}(\mathbf{R})\right)$ to itself defined by

$$
\mathbf{L}[F]=\left(\begin{array}{cc}
F_{22} & -F_{21} \\
-F_{12} & F_{11}
\end{array}\right) \quad \forall F \equiv\left(\begin{array}{ll}
F_{11} & F_{12} \\
F_{21} & F_{22}
\end{array}\right) \in \mathcal{C}^{m, \alpha}\left(\operatorname{cl} \Omega, M_{2}(\mathbf{R})\right),
$$

and let $I$ be the identity map in $\mathcal{C}^{m, \alpha}\left(\operatorname{cl} \Omega, M_{2}(\mathbf{R})\right)$. Then the following hold. 
(i) $\mathbf{L} \circ \mathbf{L}=I$,

(ii) $(I-\mathbf{L})[F]=0$ if and only if $F_{11}=F_{22}, F_{12}=-F_{21}$.

Remark 3.12. With the same notation of Lemma 3.11, let $G \equiv$ $G_{1}+i G_{2} \in \mathcal{C}^{m+1, \alpha}(\operatorname{cl} \Omega, \mathbf{C})$. Then both the first row and the first column of the $2 \times 2$ matrix

$$
\frac{1}{2}(I-\mathbf{L})[D G]
$$

equal $(\operatorname{Re} \bar{\partial} G, \operatorname{Im} \bar{\partial} G)$. Furthermore, we have $(I-\mathbf{L})[D G]=0$ in $\Omega$ if and only if $G_{1}+i G_{2}$ is holomorphic in $\Omega$.

Thus we have the following.

Proposition 3.13. Let $m \in \mathbf{N} \backslash\{0\}, \alpha \in] 0,1\left[, \Psi_{0} \in \mathcal{C}^{m, \alpha}(\operatorname{cl} \mathbf{D}, \mathbf{C}) \cap\right.$ $\mathcal{A}$. Let $\mathcal{W}_{\Psi_{0}}$ be the neighborhood of $\Psi_{0}$ in $\mathcal{C}^{m, \alpha}(\mathrm{cl} \mathbf{D}, \mathbf{C}) \cap \mathcal{A}$ of Lemma 3.7. Let $(\Psi, f) \in \mathcal{W}_{\Psi_{0}} \times \mathcal{C}_{*}^{m, \alpha}(\partial \mathbf{D}, \mathbf{C})$. The pair $\left(T^{+}, \tilde{T}\right) \in$ $\left(\mathcal{C}^{m, \alpha}(\mathrm{cl} \mathbf{D}, \mathbf{C})\right)^{2}$ satisfies the boundary value problem (3.9) if and only if the same pair satisfies the following boundary value problem

$$
\begin{cases}(I-\mathbf{L})\left[D T^{+}(\cdot) \cdot(D \Psi(\cdot))^{-1}\right]=0 & \text { in } \mathbf{D}, \\ (I-\mathbf{L})\left[D \tilde{T}(\cdot) \cdot(D \mathbf{G}[\Psi](\cdot))^{-1}\right]=0 & \text { in } \mathbf{D}, \\ T^{+}-\tilde{T}=f & \text { on } \partial \mathbf{D}, \\ \tilde{T}(0)=0 . & \end{cases}
$$

In particular, problem (3.14) associated to $(\Psi, f)$ has a unique solution $\left(T^{+}, \tilde{T}\right)$ in $\left(\mathcal{C}^{m, \alpha}(\operatorname{cl} \mathbf{D}, \mathbf{C})\right)^{2}$, and $T^{+}=\mathbf{S}^{+}[\Psi / \partial \mathbf{D}, f] \circ \Psi$.

Proof. By Remark 3.12, condition $\bar{\partial}\left[T^{+} \circ \Psi^{(-1)}\right]=0$ can be rewritten as

$$
(I-\mathbf{L})\left[D\left(T^{+} \circ \Psi^{(-1)}\right)\right]=0 \quad \text { in } \Psi(\mathbf{D}) .
$$

By taking the composition of both sides of (3.15) with $\Psi$, one obtains the first equation of (3.14). The second equation can be obtained similarly. Then we conclude by Proposition 3.8.

Our strategy is now to recast (3.14) in a form suitable for the application of the implicit function theorem. We note that the application of the implicit function theorem normally involves difficulties of 
two types. The first type of difficulty is concerned with showing the regularity of the nonlinear operators involved and with this respect we know that all of the operators appearing in (3.14) are easily seen to be real analytic, cf., Lemmas 2.3 (iii), (iv) and 3.7. The second type of difficulty is inherent with the unique solvability of the linearized problem. Although the latter type of difficulty still remains, we note that our approach has completely annihilated all the difficulties of "nonlinear type." A direct approach to show the analyticity of $(\phi, f) \rightarrow \int_{\partial \mathbf{D}} f(t) \phi^{\prime}(t) /(\phi(t)-\phi(\cdot)) d t$ would, instead, have to deal with difficulties of nonlinear type aggravated by the presence of a singular integral. Thus we now prove the following.

Theorem 3.16. Let $m \in \mathbf{N} \backslash\{0\}, \alpha \in] 0,1[$. Then the nonlinear operator defined by

$$
\mathbf{T}^{+}[\Psi, f] \equiv \mathbf{S}^{+}[\Psi / \partial \mathbf{D}, f] \circ \Psi
$$

is real analytic from $\left(\mathcal{C}^{m, \alpha}(\operatorname{cl} \mathbf{D}, \mathbf{C}) \cap \mathcal{A}\right) \times \mathcal{C}_{*}^{m, \alpha}(\partial \mathbf{D}, \mathbf{C})$ to $\mathcal{C}^{m, \alpha}(\operatorname{cl} \mathbf{D}, \mathbf{C})$.

Proof. Let $\left(\Psi_{0}, f_{0}\right) \in\left(\mathcal{C}^{m, \alpha}(\operatorname{cl} \mathbf{D}, \mathbf{C}) \cap \mathcal{A}\right) \times \mathcal{C}_{*}^{m, \alpha}(\partial \mathbf{D}, \mathbf{C})$. Let $\mathcal{W}_{\Psi_{0}}$ be the neighborhood of $\Psi_{0}$ of Lemma 3.7. We now prove the analyticity of $\mathbf{T}^{+}$by analyzing problem (3.14) for $(\Psi, f)$ in $\mathcal{W}_{\Psi_{0}} \times \mathcal{C}_{*}^{m, \alpha}(\partial \mathbf{D}, \mathbf{C})$.

With the notation of Lemma 3.11, we set

$$
\mathcal{V}^{r, \alpha} \equiv\left\{V \in \mathcal{C}^{r, \alpha}\left(\operatorname{cl} \mathbf{D}, M_{2}(\mathbf{R})\right):(I+\mathbf{L})[V]=0 \text { in } \operatorname{cl} \mathbf{D}\right\}
$$

for all $r \in \mathbf{N} . \mathcal{V}^{r, \alpha}$ is obviously a closed subspace of the Banach space $\mathcal{C}^{r, \alpha}\left(\operatorname{cl} \mathbf{D}, M_{2}(\mathbf{R})\right)$. To recast problem (3.14) in the form of a nonlinear operator equation, we define the operator $\Lambda$ of $\mathcal{W}_{\Psi_{0}} \times \mathcal{C}_{*}^{m, \alpha}(\partial \mathbf{D}, \mathbf{C}) \times$ $\left(\mathcal{C}^{m, \alpha}(\operatorname{cl} \mathbf{D}, \mathbf{C})\right)^{2}$ to $\left(\mathcal{V}^{m-1, \alpha}\right)^{2} \times \mathcal{C}_{*}^{m, \alpha}(\partial \mathbf{D}, \mathbf{C}) \times \mathbf{C}$ by means of the following equality

$$
\begin{aligned}
\Lambda\left[\Psi, f, T^{+}, \tilde{T}\right] \equiv & \left((I-\mathbf{L})\left[D T^{+}(\cdot) \cdot(D \Psi(\cdot))^{-1}\right],\right. \\
& \left.(I-\mathbf{L})\left[D \tilde{T}(\cdot) \cdot(D \mathbf{G}[\Psi](\cdot))^{-1}\right], T^{+}-\tilde{T}-f, \tilde{T}(0)\right) .
\end{aligned}
$$

By Proposition 3.13, we have

$$
\Lambda\left[\Psi, f, T^{+}, \tilde{T}\right]=0 \quad \text { if and only if }\left(T^{+}, \tilde{T}\right) \text { satisfies }(3.9) .
$$


We now apply the implicit function theorem, cf., Prodi and Ambrosetti [21, Theorem 11.6] or Berger [1, p. 134] to the operator equation (3.18). By the real analyticity of the real multi-linear continuous operators and by Lemmas 2.3 (iii), (iv) and 3.7, the operator $\Lambda$ is real analytic. Furthermore, $\Lambda$ is defined between an open subset of a Banach space and a Banach space. Let $\left(T_{0}^{+}, \tilde{T}_{0}\right)$ be the solution of $(3.9)$ corresponding to the pair $\left(\Psi_{0}, f_{0}\right)$. Then all we have to show is that the differential $d_{\left(T^{+}, \tilde{T}\right)} \Lambda\left[\Psi_{0}, f_{0}, T_{0}^{+}, \tilde{T}_{0}\right]$ of the affine map $\left(T^{+}, \tilde{T}\right) \rightarrow$ $\Lambda\left[\Psi_{0}, f_{0}, T^{+}, \tilde{T}\right]$ is a real linear homeomorphism of $\left(\mathcal{C}^{m, \alpha}(\operatorname{cl} \mathbf{D}, \mathbf{C})\right)^{2}$ onto $\left(\mathcal{V}^{m-1, \alpha}\right)^{2} \times \mathcal{C}_{*}^{m, \alpha}(\partial \mathbf{D}, \mathbf{C}) \times \mathbf{C}$. Since $d_{\left(T^{+}, \tilde{T}\right)} \Lambda\left[\Psi_{0}, f_{0}, T_{0}^{+}, \tilde{T}_{0}\right]$ is real linear and continuous, by the open mapping theorem it suffices to show that for all $\left(V_{*}^{+}, \tilde{V}_{*}, g, c\right) \in\left(\mathcal{V}^{m-1, \alpha}\right)^{2} \times \mathcal{C}_{*}^{m, \alpha}(\partial \mathbf{D}, \mathbf{C}) \times \mathbf{C}$ there exists a unique pair $\left(W_{*}^{+}, \tilde{W}_{*}\right) \in\left(\mathcal{C}^{m, \alpha}(\mathrm{cl} \mathbf{D}, \mathbf{C})\right)^{2}$ satisfying

$$
\begin{cases}(I-\mathbf{L})\left[D W_{*}^{+}(\cdot) \cdot\left(D \Psi_{0}(\cdot)\right)^{-1}\right]=V_{*}^{+} & \text {in } \mathbf{D} \\ (I-\mathbf{L})\left[D \tilde{W}_{*}(\cdot) \cdot\left(D \mathbf{G}\left[\Psi_{0}\right](\cdot)\right)^{-1}\right]=\tilde{V}_{*} & \text { in } \mathbf{D} \\ W_{*}^{+}-\tilde{W}_{*}=g & \text { on } \partial \mathbf{D} \\ \tilde{W}_{*}(0)=c & \end{cases}
$$

By composing the first equation of (3.19) with $\Psi_{0}^{(-1)}$ and the second with $\mathbf{G}\left[\Psi_{0}\right]^{(-1)}$, system (3.19) can be rewritten as

$$
\begin{cases}(I-\mathbf{L})\left[D\left(W_{*}^{+} \circ \Psi_{0}^{(-1)}\right)\right]=V_{*}^{+} \circ \Psi_{0}^{(-1)} & \text { in } \Psi_{0}(\mathbf{D}), \\ (I-\mathbf{L})\left[D\left(\tilde{W}_{*} \circ \mathbf{G}\left[\Psi_{0}\right]^{(-1)}\right)\right]=\tilde{V}_{*} \circ \mathbf{G}\left[\Psi_{0}\right]^{(-1)} & \text { in } \mathbf{G}\left[\Psi_{0}\right](\mathbf{D}), \\ W_{*}^{+}-\tilde{W}_{*}=g & \text { on } \partial \mathbf{D}, \\ \tilde{W}_{*}(0)=c . & \end{cases}
$$

Clearly,

$$
\Psi_{0}^{(-1)}(z)=\mathbf{G}\left[\Psi_{0}\right]^{(-1)}\left(\frac{1}{z-\Psi_{0}(0)}\right), \quad \forall z \in \Psi_{0}(\partial \mathbf{D}) .
$$

Now we set

$$
\begin{aligned}
V^{+} & \equiv \text { first row of } V_{*}^{+} \circ \Psi_{0}^{(-1)} / 2, \\
\tilde{V} & \equiv \text { first row of } \tilde{V}_{*} \circ \mathbf{G}\left[\Psi_{0}\right]^{(-1)} / 2 .
\end{aligned}
$$

Then, in view of Remark 3.12 and of Lemma 2.3 (v) and (vi), the existence and unique solvability in $\left(\mathcal{C}^{m, \alpha}(\mathrm{cl} \mathbf{D}, \mathbf{C})\right)^{2}$ of problem (3.19) for all 
$\left(V_{*}^{+}, \tilde{V}_{*}, g, c\right) \in\left(\mathcal{V}^{m-1, \alpha}\right)^{2} \times \mathcal{C}_{*}^{m, \alpha}(\partial \mathbf{D}, \mathbf{C}) \times \mathbf{C}$ is equivalent to existence and unique solvability in $\mathcal{C}^{m, \alpha}\left(\operatorname{cl} \Psi_{0}(\mathbf{D}), \mathbf{C}\right) \times \mathcal{C}^{m, \alpha}\left(\operatorname{cl~G}\left[\Psi_{0}\right](\mathbf{D}), \mathbf{C}\right)$ of the following linear boundary value problem

$$
\begin{cases}\bar{\partial}\left[W_{*}^{+} \circ \Psi_{0}^{(-1)}\right]=V^{+} & \text {in } \Psi_{0}(\mathbf{D}) \\ \bar{\partial}\left[\tilde{W}_{*} \circ \mathbf{G}\left[\Psi_{0}\right]^{(-1)}\right]=\tilde{V} & \text { in } \mathbf{G}\left[\Psi_{0}\right](\mathbf{D}) \\ W_{*}^{+}-\tilde{W}_{*}=g & \text { on } \partial \mathbf{D} \\ \tilde{W}_{*}(0)=c, & \end{cases}
$$

for all $\left(V^{+}, \tilde{V}, g, c\right) \in \mathcal{C}^{m-1, \alpha}\left(\operatorname{cl} \Psi_{0}(\mathbf{D}), \mathbf{C}\right) \times \mathcal{C}^{m-1, \alpha}\left(\operatorname{cl} \mathbf{G}\left[\Psi_{0}\right](\mathbf{D}), \mathbf{C}\right) \times$ $\mathcal{C}_{*}^{m, \alpha}(\partial \mathbf{D}, \mathbf{C}) \times \mathbf{C}$. By Proposition 3.8, system (3.21) has at most one solution. We now consider the existence. Let $\left(V^{+}, \tilde{V}, g, c\right) \in$ $\mathcal{C}^{m-1, \alpha}\left(\operatorname{cl} \Psi_{0}(\mathbf{D}), \mathbf{C}\right) \times \mathcal{C}^{m-1, \alpha}\left(\operatorname{cl} \mathbf{G}\left[\Psi_{0}\right](\mathbf{D}), \mathbf{C}\right) \times \mathcal{C}_{*}^{m, \alpha}(\partial \mathbf{D}, \mathbf{C}) \times \mathbf{C}$. It is well known, cf., Vekua $\left[\mathbf{2 4}\right.$, p. 56], that there exist $U^{+} \in$ $\mathcal{C}^{m, \alpha}\left(\operatorname{cl} \Psi_{0}(\mathbf{D}), \mathbf{C}\right)$ and $\tilde{U} \in \mathcal{C}^{m, \alpha}\left(\operatorname{cl} \mathbf{G}\left[\Psi_{0}\right](\mathbf{D}), \mathbf{C}\right)$ such that

$$
\begin{cases}\bar{\partial} U^{+}=V^{+} & \text {in } \Psi_{0}(\mathbf{D}), \\ \bar{\partial} \tilde{U}=\tilde{V} & \text { in } \mathbf{G}\left[\Psi_{0}\right](\mathbf{D}) .\end{cases}
$$

By possibly subtracting a constant to $\tilde{U}$, we can assume that $\tilde{U}(0)=c$. Thus all we have to show is the existence of a pair of functions $\left(T^{+}, \tilde{T}\right) \in\left(\mathcal{C}^{m, \alpha}(\operatorname{cl} \mathbf{D}, \mathbf{C})\right)^{2}$ such that

$$
\begin{cases}\bar{\partial}\left[T^{+} \circ \Psi^{(-1)}\right]=0 & \text { in } \Psi_{0}(\mathbf{D}), \\ \bar{\partial}\left[\tilde{T} \circ \mathbf{G}\left[\Psi_{0}\right]^{(-1)}\right]=0 & \text { in } \mathbf{G}\left[\Psi_{0}\right](\mathbf{D}), \\ T^{+}-\tilde{T}=g-U^{+} \circ \Psi_{0}+\tilde{U} \circ \mathbf{G}\left[\Psi_{0}\right] & \text { on } \partial \mathbf{D}, \\ \tilde{T}(0)=0 . & \end{cases}
$$

Let $h$ be the function of $\partial \mathbf{D}$ to $\mathbf{C}$ be defined by

$$
h(z) \equiv g(z)-U^{+}\left(\Psi_{0}(z)\right)+\tilde{U}\left(\mathbf{G}\left[\Psi_{0}\right](z)\right)
$$

By Lemmas 2.8 (ii) and 2.7 (vi), we have $h \in \mathcal{C}_{*}^{m, \alpha}(\partial \mathbf{D}, \mathbf{C})$. Then Proposition 3.8 ensures the existence of a pair of solutions $\left(T^{+}, \tilde{T}\right)$ of problem (3.22).

By the previous theorem, by Lemma 2.9 and by Remark 3.6, we immediately deduce the validity of the following. 
Theorem 3.23. Let $m \in \mathbf{N} \backslash\{0\}, \alpha \in] 0,1[$. The nonlinear operator $C$ from $\left(\mathcal{C}_{*}^{m, \alpha}(\partial \mathbf{D}, \mathbf{C}) \cap \mathcal{A}_{\partial \mathbf{D}}\right) \times \mathcal{C}_{*}^{m, \alpha}(\partial \mathbf{D}, \mathbf{C})$ to $\mathcal{C}_{*}^{m, \alpha}(\partial \mathbf{D}, \mathbf{C})$ defined by

$$
C[\phi, f](\cdot)=\frac{1}{2 \pi i} \text { p.v. } \int_{\partial \mathbf{D}} \frac{f(t) \phi^{\prime}(t)}{\phi(t)-\phi(\cdot)} d t
$$

for all $(\phi, f) \in\left(\mathcal{C}_{*}^{m, \alpha}(\partial \mathbf{D}, \mathbf{C}) \cap \mathcal{A}_{\partial \mathbf{D}}\right) \times \mathcal{C}_{*}^{m, \alpha}(\partial \mathbf{D}, \mathbf{C})$ is real analytic.

4. Complex analyticity of the Cauchy integral. Let $C$ be the Cauchy integral as in Theorem 3.23. We now compute all the differentials $C^{(n)}$ of $C$ and show that $C$ is complex analytic in its domain.

Proposition 4.1. Let $m, n \in \mathbf{N} \backslash\{0\}, \alpha \in] 0,1[$. Let $C$ be the nonlinear operator of Theorem 3.23. Let $\left(\phi_{0}, f_{0}\right) \in\left(\mathcal{C}_{*}^{m, \alpha}(\partial \mathbf{D}, \mathbf{C}) \cap\right.$ $\left.\mathcal{A}_{\partial \mathbf{D}}\right) \times \mathcal{C}_{*}^{m, \alpha}(\partial \mathbf{D}, \mathbf{C})$. Then the following formulas for the real partial differentials of $C$ hold.

(i)

$$
\begin{aligned}
\frac{\partial^{n} C}{(\partial \phi)^{n}}\left[\phi_{0}, f_{0}\right]\left[h_{1}, \ldots, h_{n}\right](\cdot) & \\
& =\frac{(-1)^{n}(n-1) !}{2 \pi i} \int_{\partial \mathbf{D}} f_{0}^{\prime}(t) \prod_{i=1}^{n}\left(\frac{h_{i}(t)-h_{i}(\cdot)}{\phi_{0}(t)-\phi_{0}(\cdot)}\right) d t
\end{aligned}
$$

for all $\left(h_{1}, \ldots, h_{n}\right) \in\left(\mathcal{C}_{*}^{m, \alpha}(\partial \mathbf{D}, \mathbf{C})\right)^{n}$.

(ii)

$$
\begin{aligned}
& \frac{\partial^{n} C}{(\partial \phi)^{n-1} \partial f}\left[\phi_{0}, f_{0}\right]\left[h_{1}, \ldots, h_{n-1}, k_{n}\right](\cdot) \\
= & \begin{cases}C\left[\phi_{0}, k_{n}\right](\cdot) & \text { if } n=1, \\
\frac{(-1)^{n-1}(n-2) !}{2 \pi i} \int_{\partial \mathbf{D}} k_{n}^{\prime}(t) \prod_{i=1}^{n-1}\left(\frac{h_{i}(t)-h_{i}(\cdot)}{\phi_{0}(t)-\phi_{0}(\cdot)}\right) d t & \text { if } n \geq 2,\end{cases}
\end{aligned}
$$

for all $\left(h_{1}, \ldots, h_{n-1}, k_{n}\right) \in\left(\mathcal{C}_{*}^{m, \alpha}(\partial \mathbf{D}, \mathbf{C})\right)^{n}$. 
(iii)

$$
\begin{aligned}
\left.C^{(n)}\left[\phi_{0}, f_{0}\right]\left(h_{1}, k_{1}\right), \ldots,\left(h_{n}, k_{n}\right)\right] & \\
= & \frac{\partial^{n} C}{(\partial \phi)^{n}}\left[\phi_{0}, f_{0}\right]\left[h_{1}, \ldots, h_{n}\right] \\
& +\sum_{i=1}^{n} \frac{\partial^{n} C}{(\partial \phi)^{(n-1)} \partial f}\left[\phi_{0}, f_{0}\right]\left[h_{1}, \ldots, \widehat{h}_{i}, \ldots, h_{n}, k_{i}\right],
\end{aligned}
$$

for all $\left(h_{1}, k_{1}, \ldots, h_{n}, k_{n}\right) \in\left(\mathcal{C}_{*}^{m, \alpha}(\partial \mathbf{D}, \mathbf{C})\right)^{2 n}$. The “', symbol on a term denotes that such a term must be omitted. In particular, $C$ is complex analytic in its domain.

Proof. It clearly suffices to consider the case in which ind $\left[\phi_{0}\right]=1$. Let $R$ be the trace operator of $\mathcal{C}^{m, \alpha}(\mathrm{cl} \mathbf{D}, \mathbf{C})$ to $\mathcal{C}_{*}^{m, \alpha}(\partial \mathbf{D}, \mathbf{C})$. Let $z_{0} \in \mathbf{I}\left[\phi_{0}\right]$, and let $E, E_{\phi_{0}}, \Psi_{0}$ and $\mathcal{U}_{\phi_{0}}$ be as in Lemma 2.9. Clearly, we can assume that ind $[\phi]=1$ for all $\phi \in \mathcal{U}_{\phi_{0}}$. The operator $E_{\phi_{0}}$ is complex differentiable at all points of its domain, with differential given by the operator $E$ which satisfies $E[h]_{/ \partial \mathbf{D}}=h$ for all $h \in \mathcal{C}_{*}^{m, \alpha}(\partial \mathbf{D}, \mathbf{C})$. We now compute the Taylor expansion of the real analytic operator $C\left[\cdot, f_{0}\right]$ at $\phi_{0}$. By the definition of $\mathbf{T}^{+}$, cf. (3.17), and of $C$ and by the Plemelj formula (3.4), we have

$$
C\left[\phi, f_{0}\right]=-\frac{1}{2} f_{0}+R\left[\mathbf{T}^{+}\left[E_{\phi_{0}}[\phi], f_{0}\right]\right] \quad \text { on } \partial \mathbf{D},
$$

for all $\phi \in \mathcal{U}_{\phi_{0}}$. Since $R$ is linear, it suffices to find the Taylor expansion at $\Psi_{0} \equiv E_{\phi_{0}}\left[\phi_{0}\right]$ of the operator $\mathbf{T}^{+}\left[\cdot, f_{0}\right]$ of $\mathcal{C}^{m, \alpha}(\operatorname{cl} \mathbf{D}, \mathbf{C}) \cap \mathcal{A}$ to $\mathcal{C}^{m, \alpha}(\operatorname{cl} \mathbf{D}, \mathbf{C})$. We set

$$
\left.\mathbf{D}_{\rho}=\left\{\left(x_{1}, x_{2}\right) \in \mathbf{R}^{2}:\left|\left(x_{1}, x_{2}\right)\right|<\rho\right\} \quad \forall \rho \in\right] 0,1[,
$$

and we denote by $R_{\rho}$ the restriction operator of $\mathcal{C}^{m, \alpha}(\operatorname{cl} \mathbf{D}, \mathbf{C})$ to $\mathcal{C}^{m, \alpha}\left(\mathrm{cl} \mathbf{D}_{\rho}, \mathbf{C}\right)$. Then we have that

$$
R_{\rho} \circ \mathbf{T}^{+}\left[\Psi, f_{0}\right](w)=\frac{\operatorname{ind}[\Psi / \partial \mathbf{D}]}{2 \pi i} \int_{\partial \mathbf{D}} \frac{f_{0}(t) \Psi^{\prime}(t)}{\Psi(t)-\Psi(w)} d t \quad \forall w \in \operatorname{cl} \mathbf{D}_{\rho},
$$

for all $\Psi \in \mathcal{C}^{m, \alpha}(\operatorname{cl} \mathbf{D}, \mathbf{C}) \cap \mathcal{A}$ and for all $\left.\rho \in\right] 0,1[$. Now we note that, by Theorem 3.16, $R_{\rho} \circ \mathbf{T}^{+}\left[\cdot, f_{0}\right]$ is real analytic on $\mathcal{C}^{m, \alpha}(\operatorname{cl} \mathbf{D}, \mathbf{C}) \cap \mathcal{A}$ 
and that by standard calculus

$$
\begin{aligned}
R_{\rho} \circ\left(\frac{\partial^{n} \mathbf{T}^{+}}{(\partial \Psi)^{n}}\left[\Psi_{0}, f_{0}\right]\right)\left[[U]^{n}\right] & =\frac{\partial^{n}}{(\partial \Psi)^{n}}\left(R_{\rho} \circ \mathbf{T}^{+}\right)\left[\Psi_{0}, f_{0}\right]\left[[U]^{n}\right] \\
& =\frac{d^{n}}{\left.(d \varepsilon)^{n}\right|_{\varepsilon=0}}\left\{R_{\rho} \circ \mathbf{T}^{+}\left[\Psi_{0}+\varepsilon U, f_{0}\right]\right\}
\end{aligned}
$$

for all $U \in \mathcal{C}^{m, \alpha}(\operatorname{cl} \mathbf{D}, \mathbf{C})$. Since $\mathcal{C}^{m, \alpha}(\operatorname{cl} \mathbf{D}, \mathbf{C})$ is continuously embedded in $\mathcal{C}^{0}(\mathrm{cl} \mathbf{D}, \mathbf{C})$ and since the integral in (4.3) is not singular when $w \in \operatorname{cl} \mathbf{D}_{\rho}$, then by a standard result on the differentiation of integrals depending on a parameter and by a straightforward computation, we obtain that

$$
\begin{aligned}
& \frac{d^{n}}{\left.(d \varepsilon)^{n}\right|_{\varepsilon=0}}\left\{R_{\rho} \circ \mathbf{T}^{+}\left[\Psi_{0}+\varepsilon U, f_{0}\right]\right\}(w) \\
& =\frac{1}{2 \pi i} \int_{\partial \mathbf{D}} \frac{d^{n}}{(d \varepsilon)^{n} \mid \varepsilon=0}\left\{\frac{f_{0}(t)\left(\Psi_{0}^{\prime}+\varepsilon U^{\prime}\right)(t)}{\left(\Psi_{0}+\varepsilon U\right)(t)-\left(\Psi_{0}+\varepsilon U\right)(w)}\right\} d t \\
& =\frac{(-1)^{n-1} n !}{2 \pi i} \int_{\partial \mathbf{D}}\left(\frac{U(t)-U(w)}{\Psi_{0}(t)-\Psi_{0}(w)}\right)^{n-1} \\
& \quad \cdot\left\{-\frac{f_{0}(t) \Psi_{0}^{\prime}(t)(U(t)-U(w))}{\left(\Psi_{0}(t)-\Psi_{0}(w)\right)^{2}}+\frac{f_{0}(t) U^{\prime}(t)}{\Psi_{0}(t)-\Psi_{0}(w)}\right\} d t \\
& =\frac{(-1)^{n-1}(n-1) !}{2 \pi i} \int_{\partial \mathbf{D}} f_{0}(t) \frac{d}{d t}\left\{\left(\frac{U(t)-U(w)}{\Psi_{0}(t)-\Psi_{0}(w)}\right)^{n}\right\} d t \\
& =\frac{(-1)^{n}(n-1) !}{2 \pi i} \int_{\partial \mathbf{D}} f_{0}^{\prime}(t)\left(\frac{U(t)-U(w)}{\Psi_{0}(t)-\Psi_{0}(w)}\right)^{n} d t
\end{aligned}
$$

for all $w \in \mathrm{cl} \mathbf{D}_{\rho}$, where the last equality follows by integration by parts. Then, if $w \in \mathbf{D}$, we can choose $\rho$ such that $|w|<\rho<1$ and, by (4.4), we obtain

$$
\begin{aligned}
& \frac{\partial^{n} \mathbf{T}^{+}}{(\partial \Psi)^{n}}\left[\Psi_{0}, f_{0}\right]\left[[U]^{n}\right](w) \\
& \quad=\frac{(-1)^{n}(n-1) !}{2 \pi i} \int_{\partial \mathbf{D}} f_{0}^{\prime}(t)\left(\frac{U(t)-U(w)}{\Psi_{0}(t)-\Psi_{0}(w)}\right)^{n} d t
\end{aligned}
$$

Since $U \equiv U_{1}+i U_{2}$ belongs to $\mathcal{C}^{1}(\operatorname{cl} \mathbf{D}, \mathbf{C})$, by Lemma 2.4 (i) and by the mean value inequality, we obtain

$$
\left|\frac{U(t)-U(w)}{\Psi_{0}(t)-\Psi_{0}(w)}\right| \leq \frac{|U(t)-U(w)|}{\left.l \mid \Psi_{0}\right]|t-w|} \leq \frac{\sum_{1 \leq h, j \leq 2} \sup _{\mathrm{cl} \mathbf{D}}\left|\partial U_{h} / \partial x_{j}\right|}{l\left[\Psi_{0}\right]},
$$


for all $(t, w) \in \partial \mathbf{D} \times \operatorname{cl} \mathbf{D}$ with $t \neq w$. Then, by the theorem of continuity of integrals depending on a parameter, the righthand side of (4.5) depends continuously on $w \in \mathrm{cl} \mathbf{D}$ and then equality (4.5) holds for all $w \in \operatorname{cl} \mathbf{D}$. By (4.6), the equality

$$
\begin{gathered}
\mathbf{H}\left[U_{1}, \ldots, U_{n}\right](w) \equiv \frac{(-1)^{n}(n-1) !}{2 \pi i} \int_{\partial \mathbf{D}} f_{0}^{\prime}(t) \prod_{i=1}^{n}\left(\frac{U_{i}(t)-U_{i}(w)}{\Psi_{0}(t)-\Psi_{0}(w)}\right) d t \\
\forall w \in \operatorname{cl} \mathbf{D},
\end{gathered}
$$

for all $\left(U_{1}, \ldots, U_{n}\right) \in\left(\mathcal{C}^{m, \alpha}(\operatorname{cl} \mathbf{D}, \mathbf{C})\right)^{n}$, defines a complex $n$-linear symmetric map of $\left(\mathcal{C}^{m, \alpha}(\operatorname{cl} \mathbf{D}, \mathbf{C})\right)^{n}$ to $\mathcal{C}^{0}(\operatorname{cl} \mathbf{D}, \mathbf{C})$. Clearly,

$$
\mathbf{H}\left[[U]^{n}\right]=\frac{\partial^{n} \mathbf{T}^{+}}{(\partial \Psi)^{n}}\left[\Psi_{0}, f_{0}\right]\left[[U]^{n}\right]
$$

for all $\left.U \in \mathcal{C}^{m, \alpha}(\operatorname{cl} \mathbf{D}, \mathbf{C})\right)$. Since $\mathcal{C}^{m, \alpha}(\operatorname{cl} \mathbf{D}, \mathbf{C})$ is continuously embedded in $\mathcal{C}^{0}(\operatorname{cl} \mathbf{D}, \mathbf{C})$ and both $\mathbf{H}$ and $\left(\partial^{n} \mathbf{T}^{+} /(\partial \Psi)^{n}\right)\left[\Psi_{0}, f_{0}\right]$ are real $n$-linear symmetric maps which coincide on the diagonal of $\left(\mathcal{C}^{m, \alpha}(\mathrm{cl} \mathbf{D}, \mathbf{C})\right)^{n}$, we must have

$$
\mathbf{H}=\frac{\partial^{n} \mathbf{T}^{+}}{(\partial \Psi)^{n}}\left[\Psi_{0}, f_{0}\right]
$$

and accordingly $\left(\partial^{n} \mathbf{T}^{+} /(\partial \Psi)^{n}\right)\left[\Psi_{0}, f_{0}\right]$ is a complex $n$-linear map. By using the chain rule combined with the properties of the map $E_{\phi_{0}}$, we obtain statement (i). The linearity of $C$ in the variable $f$ implies the validity of statement (ii) and of equality

$$
\frac{\partial^{j+2} C}{(\partial \phi)^{j}(\partial f)^{2}}[\phi, f]=0
$$

for all $(\phi, f)$ in the domain of $C$ and for all $j \in \mathbf{N}$. We obtain statement (iii) by statements (i), (ii), (4.7) and by induction on $n$. Since for all $r \in \mathbf{N} \backslash\{0\}, C^{(r)}\left[\phi_{0}, f_{0}\right]$ is a complex $r$-linear symmetric map from $\left(\mathcal{C}_{*}^{m, \alpha}(\partial \mathbf{D}, \mathbf{C})\right)^{r}$ to $\mathcal{C}_{*}^{m, \alpha}(\partial \mathbf{D}, \mathbf{C})$, Theorem 3.23 implies that $C$ is a complex analytic operator.

Now we restate Theorem 3.23 by using a domain of integration more general than $\partial \mathbf{D}$. 
Corollary 4.8. Let $m \in \mathbf{N} \backslash\{0\}, \alpha \in] 0,1\left[\right.$. Let $\phi \in \mathcal{C}_{*}^{m, \alpha}(\partial \mathbf{D}, \mathbf{C}) \cap$ $\mathcal{A}_{\partial \mathbf{D}}, L=\phi(\partial \mathbf{D})$. The set of $g \in \mathcal{C}_{*}^{1}(L, \mathbf{C})$ which are injective and satisfy condition $g^{\prime}(\xi) \neq 0$ for all $\xi \in L$ coincides with $\mathcal{A}_{L}$ and is open in $\mathcal{C}_{*}^{1}(L, \mathbf{C})$. The nonlinear operator of $\left(\mathcal{C}_{*}^{m, \alpha}(L, \mathbf{C}) \cap \mathcal{A}_{L}\right) \times \mathcal{C}_{*}^{m, \alpha}(L, \mathbf{C})$ to $\mathcal{C}_{*}^{m, \alpha}(L, \mathbf{C})$ defined by

$$
\begin{gathered}
C_{L}[\gamma, f](\cdot) \equiv \frac{1}{2 \pi i} \text { p.v. } \int_{L} \frac{f(t) \gamma^{\prime}(t)}{\gamma(t)-\gamma(\cdot)} d t \\
\forall(\gamma, f) \in\left(\mathcal{C}_{*}^{m, \alpha}(L, \mathbf{C}) \cap \mathcal{A}_{L}\right) \times \mathcal{C}_{*}^{m, \alpha}(L, \mathbf{C}),
\end{gathered}
$$

where we understand that the line integral is computed with respect to the parametrization $\phi\left(e^{i \theta}\right), \theta \in[0,2 \pi]$, is complex analytic. The partial differentials of $C_{L}$ can be obtained by those of $C_{\partial \mathbf{D}}=C$ by replacing the integration on $\partial \mathbf{D}$ with an integration on $L$.

Proof. By simple computations and by Lemma 2.7 (ii), it follows that $K=\phi(\partial \mathbf{D})$ satisfies the assumptions on $K$ of conditions (i) and (ii) of Lemma 2.5. Accordingly, the conclusions of Lemma 2.5 (i) and (ii) hold for $K=\phi(\partial \mathbf{D})$. Let $\mathbf{T}_{\phi}$ be the complex linear homeomorphism of $\mathcal{C}_{*}^{m, \alpha}(L, \mathbf{C})$ to $\mathcal{C}_{*}^{m, \alpha}(\partial \mathbf{D}, \mathbf{C})$ of Lemma 2.7 (viii). Clearly,

$$
C_{L}[\gamma, f]=\mathbf{T}_{\phi}^{(-1)}\left[C\left[\mathbf{T}_{\phi}[\gamma], \mathbf{T}_{\phi}[f]\right]\right]
$$

for $(\gamma, f) \in\left(\mathcal{C}_{*}^{m, \alpha}(L, \mathbf{C}) \cap \mathcal{A}_{L}\right) \times \mathcal{C}_{*}^{m, \alpha}(L, \mathbf{C})$. Then the chain rule yields the conclusion.

We now show that the formal expansion of the Cauchy kernel with respect to the curve $\phi$ studied by Tran-Oberlé $[\mathbf{2 2}]$ for graph curves in $\mathbf{C}$ around the inclusion map of $\mathbf{R}$ in $\mathbf{C}$ gives, in our setting, the Taylor series of the Cauchy operator as a function of the contour $\phi$. As a consequence, we deduce the validity of a result of Coifman and Meyer $[\mathbf{5}$, p. 10] in our Schauder space setting and for curves which are not necessarily arc-length parametrized.

Corollary 4.9. Let $m \in \mathbf{N} \backslash\{0\}, \alpha \in] 0,1[$. Let $C$ be the nonlinear operator of Theorem 3.23. The nonlinear operator $\tilde{C}$ from $\mathcal{C}_{*}^{m, \alpha}(\partial \mathbf{D}, \mathbf{C}) \cap \mathcal{A}_{\partial \mathbf{D}}$ to $\mathcal{L}_{\mathbf{C}}\left(\mathcal{C}_{*}^{m, \alpha}(\partial \mathbf{D}, \mathbf{C}), \mathcal{C}_{*}^{m, \alpha}(\partial \mathbf{D}, \mathbf{C})\right)$ defined by

$$
\tilde{C}[\phi] \equiv C[\phi, \cdot]
$$


is complex analytic. Furthermore, if $\phi_{0} \in \mathcal{C}_{*}^{m, \alpha}(\partial \mathbf{D}, \mathbf{C}) \cap \mathcal{A}_{\partial \mathbf{D}}$ and if we denote the integral operators $f \mapsto \int_{\partial \mathbf{D}} f(t) k(\cdot, t) d t$ and $f \mapsto$ p.v. $\int_{\partial \mathbf{D}} f(t) k(\cdot, t) d t$ associated to a given complex-valued function $k(\cdot, \cdot)$ defined on $\left\{(s, t) \in(\partial \mathbf{D})^{2}: s \neq t\right\}$ by $k(\cdot, t) d t$ and p.v. $k(\cdot, t) d t$, respectively, we have the following Taylor expansion which has radius of convergence greater than or equal to $\tilde{r} \equiv \sup \{r>0$ : $\left.\mathrm{cl}_{\mathcal{C}_{*}^{m, \alpha}(\partial \mathbf{D}, \mathbf{C})} \mathcal{B}\left(\phi_{0}, r\right) \subseteq \mathcal{C}_{*}^{m, \alpha}(\partial \mathbf{D}, \mathbf{C}) \cap \mathcal{A}_{\partial \mathbf{D}}\right\}$, where $\mathcal{B}\left(\phi_{0}, r\right) \equiv\{\phi \in$ $\left.\mathcal{C}_{*}^{m, \alpha}(\partial \mathbf{D}, \mathbf{C}):\left\|\phi-\phi_{0}\right\|_{\mathcal{C}_{*}^{m, \alpha}(\partial \mathbf{D}, \mathbf{C})}<r\right\}:$

$$
\begin{aligned}
\frac{1}{2 \pi i} \text { p.v. } \frac{\phi_{0}^{\prime}(t)+}{\phi_{0}(t)-\phi_{0}(t)}+ & h(t)-h(\cdot) \\
= & \frac{1}{2 \pi i} \text { p.v. } \frac{\phi_{0}^{\prime}(t)}{\phi_{0}(t)-\phi_{0}(\cdot)} d t \\
& \quad+\sum_{n=1}^{\infty} \frac{(-1)^{n-1}}{2 \pi i n} \frac{d}{d t}\left\{\left(\frac{h(t)-h(\cdot)}{\phi_{0}(t)-\phi_{0}(\cdot)}\right)^{n}\right\} d t
\end{aligned}
$$

for all $h \in \mathcal{C}_{*}^{m, \alpha}(\partial \mathbf{D}, \mathbf{C})$ such that $\|h\|_{\mathcal{C}_{*}^{m, \alpha}(\partial \mathbf{D}, \mathbf{C})}<r$.

Proof. We first observe that $C$ is linear in its second variable. Accordingly, if $f_{0}$ is an arbitrary but fixed element of $\mathcal{C}_{*}^{m, \alpha}(\partial \mathbf{D}, \mathbf{C})$, we have

$$
\tilde{C}[\phi]=\frac{\partial C}{\partial f}\left[\phi, f_{0}\right]
$$

for all $\phi \in \mathcal{C}_{*}^{m, \alpha}(\partial \mathbf{D}, \mathbf{C}) \cap \mathcal{A}_{\partial \mathbf{D}}$. Then the analyticity of $\tilde{C}$ follows from that of $C$. A simple computation based on the Hölder continuity of $\phi_{0}^{\prime}, h^{\prime}$ and on Lemmas 2.5 (i) and 2.7 (i) shows that there exists a constant $c>0$ depending only on $h$ and $\phi_{0}$ such that $\left|(d / d t)\left[\left(h(t)-h\left(t_{0}\right)\right) /\left(\phi_{0}(t)-\phi_{0}\left(t_{0}\right)\right)\right]\right| \leq\left(c /\left|t-t_{0}\right|^{1-\alpha}\right)$ for all $t, t_{0} \in \partial \mathbf{D}, t \neq t_{0}$, see also $\mathrm{Lu}[\mathbf{1 8}, \mathrm{p} .20]$, and that accordingly the integral $\int_{\partial \mathbf{D}} f(t)(d / d t)\left[\left(h(t)-h\left(t_{0}\right)\right) /\left(\phi_{0}(t)-\phi_{0}\left(t_{0}\right)\right)\right] d t$ exists in the sense of Lebesgue for all $f \in \mathcal{C}_{*}^{0}(\partial \mathbf{D}, \mathbf{C})$ and for all $t_{0} \in \partial \mathbf{D}$. By Proposition 4.1 (ii) and by integration by parts, we obtain the validity of the Taylor expansion of the statement in a ball $\mathcal{B}\left(\phi_{0}, r_{1}\right)$ of sufficiently small radius $r_{1}>0$. Let $r>0$ be such that $\overline{\mathcal{B}} \equiv \operatorname{cl}_{\mathcal{C}_{*}^{m, \alpha}(\partial \mathbf{D}, \mathbf{C})} \mathcal{B}\left(\phi_{0}, r\right)$ is contained in $\mathcal{C}_{*}^{m, \alpha}(\partial \mathbf{D}, \mathbf{C}) \cap \mathcal{A}_{\partial \mathbf{D}}$. To complete the proof it suffices to show that $\tilde{C}[\cdot]$ is bounded on $\overline{\mathcal{B}}$. By a standard application of the Ascoli-Arzela theorem, the set $\overline{\mathcal{B}}$ is a compact subset of $\mathcal{C}_{*}^{1}(\partial \mathbf{D}, \mathbf{C})$. 
Then Lemma 2.5 (ii) implies that the map $l_{\partial \mathbf{D}}[\cdot]$ has a strictly positive minimum on $\overline{\mathcal{B}}$. Then, by the Privalov theorem, cf., e.g., Lu $[\mathbf{1 8}$, Theorem 3.1.1] and by standard properties of the Cauchy integral, the boundedness of $\tilde{C}[\cdot]$ on $\overline{\mathcal{B}}$ follows.

\section{REFERENCES}

1. M.S. Berger, Nonlinearity and functional analysis, Academic Press, New York, 1977.

2. A.P. Calderón, Cauchy integrals on Lipschitz curves and related operators, Proc. Natl. Acad. Sci. U.S.A. 74 (1977), 1324-1327.

3. R.R. Coifman, A. McIntosh and Y. Meyer, L'intégrale de Cauchy définit un opérateur borné sur $L^{2}$ pour les courbes lipschitziennes, Ann. of Math. 116 (1982), 361-387.

4. R.R. Coifman and Y. Meyer, L'analyse armonique non linéaire, in Proc. of a seminar held in Turin and Milan in 1982: Topics on modern harmonic analysis (De Leonida-Ricci, ed.), INDAM F. Severi, Roma, 2 1983, 707-721.

5. — Lavrentiev's curves and conformal mappings, Report No. 5, Institut Mittag-Leffler, 1983.

6. G. David, Opérateurs intégraux singuliers sur certaines courbes du plan complexe, Ann. Sci. École Norm. Sup. 4e série 17 (1984), 157-189.

7. C. Godbillon, Éléments de topologie algébrique, Hermann, Paris, 1971.

8. E. Hille and R.S. Phillips, Functional analysis and semigroups, Amer. Math. Soc. Colloq. Publ. 31 (1957).

9. W. Hurewicz and H. Wallman, Dimension theory, Princeton University Press, Princeton, 1948.

10. P. Jones, Quasiconformal mappings and extendability of functions in Sobolev spaces, Acta Math. 147 (1981), 71-88.

11. J.L. Journé, Calderón-Zygmund operators, pseudo-differential operators and the Cauchy integral of Calderón, Lecture Notes in Math. 994, Springer-Verlag, New York, 1983.

12. A. Kufner, O. John and S. Fučík, Function spaces, Noordhoff Internat. Publ., Leyden, 1977.

13. M. Lanza de Cristoforis, Properties and pathologies of the composition and inversion operators in Schauder spaces, Acc. Naz. delle Sci. detta dei XL 15 (1991), 93-109.

14. - Differentiability properties of an abstract autonomous composition operator, 1996 (submitted).

15. - A functional decomposition theorem for the conformal representation, J. Math. Soc. Japan 49 (1997), 759-780.

16. M. Lanza de Cristoforis and S.S. Antman, The large deformation of nonlinearly elastic tubes in two-dimensional flows, SIAM J. Math. Anal. 22 (1991), 1193-1221. 
17. M. Lanza de Cristoforis and S.V. Rogosin, Analyticity of a nonlinear operator associated to the conformal representation in Schauder spaces. An integral equation approach, 1997 (submitted).

18. J.K. Lu, Boundary value problems for analytic functions, World Scientific, Singapore, 1993.

19. Y. Meyer and R.R. Coifman, Ondelettes et opérateurs, vol. III, Éditeurs des Sciences et des Arts, Hermann, Paris, 1991.

20. N.I. Muskhelishvili, Singular integral equations, Groningen-Holland, 1953.

21. G. Prodi and A. Ambrosetti, Analisi non lineare, Editrice tecnico scientifica, Pisa, 1973.

22. C. Tran-Oberlé, Analyse non linéaire de l'opérateur défini par l'intégrale de Cauchy, Bull. Soc. Math. France 117 (1989), 1-18.

23. G.M. Troianiello, Elliptic differential equations and obstacle problems, Plenum Publishing Co., New York, 1987.

24. I.N. Vekua, Generalized analytic functions, Pergamon Press, New York, 1962.

25. S. Wu, Analytic dependence of Riemann mappings for bounded domains and minimal surfaces, Comm. Pure Appl. Math. 46 (1993), 1303-1326.

Dipartimento di Matematica Pura ed Applicata, Università di Padova, Via G. Belzoni N. 7, I-35131 Padova, Italy

E-mail address: mldc@math.unipd.it

Dipartimento di Matematica Pura ed Applicata, Università di Padova, via G. Belzoni n. 7, I-35131 Padova, Italy

E-mail address: preciso@math.unipd.it 